\title{
Temporal and Spatial Patterns of Tenascin and Laminin Immunoreactivity Suggest Roles for Extracellular Matrix in Development of Gustatory Papillae and Taste Buds
}

\author{
CHARLOTTE M. MISTRETTA AND LINDA F. HAUS \\ Department of Biologic and Materials Sciences, School of Dentistry, \\ University of Michigan, Ann Arbor, MI 48109-1078
}

\begin{abstract}
Gustatory papillae are complex organs that are composed of 1) an epithelium, 2) specialized sensory cells within the epithelium (the taste buds), 3) a broad connective core, and 4) sensory innervation. During papilla development, cells in the various tissue compartments must divide, aggregate, detach, migrate, and reaggregate in relation to each other, but factors that regulate such steps are poorly understood and have not been extensively studied. All of these processes potentially require participation of the extracellular matrix. Therefore, we have studied temporal and spatial patterns of immunoreactivity for two extracellular matrix molecules, tenascin and laminin, in the developing fungiform and circumvallate papillae of fetal, perinatal, and adult sheep tongue. To determine relations of tenascin and laminin to sensory innervation, we used an antibody to growth-associated protein (GAP-43) to label growing nerves. Immunocytochemical distributions of tenascin and laminin alter during development in a manner that reflects morphogenesis rather than histologic boundaries of the taste papillae. In early fungiform papillae, tenascin immunoreactivity is very weak within the mesenchyme of the papilla core. However, there is a subsequent shift to an intense, restricted localization in the apical papilla core only-directly under taste bud-bearing regions of the papilla epithelium. In early circumvallate papillae, tenascin immunoreactivity is patchy within the papilla core and within the flanking, nongustatory papillae. Later, immunoreactivity is restricted to the perimeter of the central papilla core, under epithelium that contains developing taste buds. In fungiform and circumvallate papillae, the shift in tenascin immunolocalization is associated with periods of taste bud formation and multiplication within the papilla epithelium and with extensive branching of the sensory innervation in the papilla apex. Laminin immunoreactivity, although it is continuous throughout the basement membrane of general lingual epithelium, is interrupted in the epithelial basement membrane of early fungiform and circumvallate papillae in regions where taste buds are forming. The breaks are large in young fetuses, when taste buds first develop, and are evidenced later as punctate disruptions. Heparan sulfate proteoglycan immunoreactivity confirms that these are basement membrane discontinuities. GAP-43 label coincides with innervation of the papilla core and is most extensive in regions where tenascin immunoreactivity is weak or absent. GAP-43 immunoreactivity is also found in early taste buds: Later, it is extensive within more mature multiple taste buds, presumably in relation to synaptogenesis. We propose that tenascin has a role in promoting deadhesion of cells in the papilla epithelium during periods of taste bud formation and multiplication. Discontinuities in
\end{abstract}

\footnotetext{
Accepted June 30, 1995

Address reprint requests to Dr. Charlotte M. Mistretta, Room 6228 , School of Dentistry, University of Michigan, Ann Arbor, MI 48109-1078
} 
the epithelial basement membrane under developing taste buds, indicated with laminin and heparan sulfate proteoglycan immunoreactivity, may interact to facilitate taste bud morphogenesis and multiplication, to permit access of papilla innervation to the forming taste buds, and/or to allow epithelial/mesenchymal interactions during papilla and taste bud development. 1996 Wiley-Liss, Inc.

Indexing terms: fungiform papilla, circumvallate papilla, morphogenesis, GAP-43, basement membrane

Taste buds in the mammalian tongue reside in specialized epithelial organs, the gustatory papillae, and are never found in the general lingual epithelium. Although there is knowledge about the time course and morphological events of papilla development, there is virtually no understanding about cell and molecular mechanisms that direct papilla formation, growth, and maintenance (Mistretta, 1991, 1993; Mistretta and Hill, 1995). Because the extracellular matrix has demonstrated morphogenetic functions in several organ systems, we have determined the distribution of extracellular matrix molecules during development of gustatory papillae in fetal, perinatal, and adult sheep tongue.

The extracellular matrix is an essential component of the cell environment that provides structural support, acts as a molecular barrier and filter, and regulates cell behavior (Adams and Watt, 1993). During development, extracellular matrix molecules exert regulatory control on processes of cell attachment, spreading, differentiation, proliferation, and polarization of cells (End and Engel, 1991). The gustatory papillae that contain taste buds on the tongue are complex organs composed of epithelium, connective tissue core, innervation, and sensory cells. During papilla development, cells in these various tissue compartments divide, aggregate, detach, move, and realign in relation to each other: all processes that potentially require extracellular matrix controls.

An extracellular matrix molecule that has been extensively studied in numerous developing systems is the large glycoprotein tenascin, which is notable for its appearance in spatially restricted patterns at specific times in the embryo and for its virtual absence from most adult tissues (ChiquetEhrismann, 1990; Erickson, 1993). Tenascin is found in the mesenchyme during periods of important tissue interactions in organogenesis (for example, during epithelial/ mesenchymal interactions) and is often localized near epithelium when cells are proliferating and migrating (Erickson and Lightner, 1988). Among the regulatory functions for tenascin are roles in development of feather, hair, kidney, lung, skin, and tooth (Aufderheide et al., 1987; Thesleff et al., 1987; Lightner et al., 1989; Koch et al., 1991; Tucker, 1991; Kaplan and Holbrook, 1994; Young et al., 1994). In addition, tenascin is implicated in regulation of peripheral nerve outgrowth and fasciculation (Chiquet, 1989; Wehrle and Chiquet, 1990).

Laminin is an essential component of basement membranes, the rigid extracellular matrix that is common to all tissues (Yurchenco and Schittny, 1990). Like tenascin, laminin is also known to have roles in cell differentiation, adhesion, migration, proliferation, and polarity (Kleinman et al., 1985; Martin and Timpl, 1987; Tryggvason, 1993). Laminin is the first extracellular matrix molecule expressed in the mammalian embryo (Martin and Timpl, 1987) and is extensive in regions of the embryo where epithelial development is occurring (Klein et al., 1988). Furthermore, laminin is a potent promoter of neurite outgrowth for neurons in culture (Clark et al., 1993; Tomaselli et al., 1993) and is thought to form a prepathway for nerve growth cone guidance in vivo (Rogers et al., 1986; Riggott and Moody, 1987).

Although tenascin, laminin, and other extracellular matrix molecules are well studied in numerous organs, there is nothing known about their possible roles in the developing taste system. Other extracellular matrix molecules will undoubtedly be important in gustatory development as well. However, we began with a study of tenascin because it has a restricted spatial and temporal distribution that is selectively present in mesenchyme surrounding developing epithelia in various organs and because data suggest that it regulates interactions between cells and the extracellular matrix to promote cell migration and differentiation (Erickson, 1993). Laminin was selected because it is a major basement membrane component with established roles in developing organs and neural systems (Tryggvason, 1993). Furthermore, tenascin and laminin have potentially contrasting roles in morphogenesis: tenascin in cell deadhesion and laminin in adhesion processes (Murphy-Ullrich et al., 1991). Therefore, we have examined fungiform and circumvallate papillae in fetal, perinatal, and adult sheep to determine the spatial and temporal immunolocation of tenascin and laminin. For an additional basement membrane component, heparan sulfate proteoglycan (HPSG) immunoreactivity also was examined. To correlate developmental patterns of extracellular matrix components with tongue and papilla innervation, we studied distributions of growing nerves using an antibody to growth associated protein-43 (GAP-43; Reynolds et al., 1991). These immunocytochemical investigations are an initial step in the study of regulatory roles for the extracellular matrix in taste development.

Sheep gustatory organs were used because there is extensive knowledge about functional and morphological development of the gustatory system in fetal, perinatal, and postnatal sheep (Bradley and Mistretta, 1973; Mistretta and Bradley, 1983a; Mistretta et al., 1988; Nagai et al., 1988; Vogt and Mistretta, 1990; Mistretta and Labyak, 1994). The sheep gustatory system has aspects of temporal development and structural characteristics that compare very well to the human system (Mistretta, 1990, 1991). Also, fetal and postnatal taste papillae in sheep are large organs with numerous taste buds and with an extensive innervation that render them especially amenable to study of extracellular matrix components. Preliminary reports of these studies have appeared in abstracts (Haus and Mistretta, 1992; Mistretta and Haus, 1992, 1993). 


\section{MATERIALS AND METHODS Animals, surgical preparation, and tissue collection}

Tissue was collected from Suffolk sheep in seven age groups: fetuses aged 49-51 days of gestation, 75-80 days of gestation, 100-102 days of gestation, 108-112 days of gestation, and 127-133 days of gestation; perinatal animals aged 141 days of gestation to 1 day postnatal (term $\sim 147$ days of gestation); and adult animals. These groups are designated 50DF, 80DF, 100DF, 110DF, 130DF, peri, and adult. Tissue from four to six animals was analyzed for each age group. In addition, we studied tongues from two fetuses at 35-40 days of gestation, a period in advance of papilla formation. Pregnant ewes were anesthetized with an intravenous injection of sodium pentobarbital $(30 \mathrm{mg} / \mathrm{kg})$, a dose that also anesthetizes the fetus (Bradley and Mistretta, 1973). Subsequent anesthetic was administered via an indwelling jugular catheter. Ewes were tracheotomized, and the fetuses were delivered by Cesarean section onto a table next to the ewe. Umbilical and placental circulation remained intact, so that the fetus was anesthetized and maintained via the ewe. At the conclusion of tissue collection, ewe and fetus received a lethal overdose of anesthetic.

Tongues from fetuses at 35-40 days of gestation and from the 50DF and $80 \mathrm{DF}$ groups were removed and placed in $0.1 \mathrm{M}$ phosphate buffer, $\mathrm{pH} 7.4$, at $4^{\circ} \mathrm{C}$, and anterior and posterior tongue tissues with fungiform and circumvallate papillae were further dissected. Animals from the remaining groups were perfused through the heart with $0.1 \mathrm{M}$ phosphate buffer before the tissue was collected. Tissue was placed in molds containing embedding medium (O.C.T.; Miles Scientific), frozen in hexane cooled by an acetone and solid carbon dioxide bath, and stored at $-80^{\circ} \mathrm{C}$ until it was used for sectioning. Sections were cut at $10 \mu \mathrm{m}$ on a Reichert-Jung Cryocut 1800 cryostat in transverse or sagittal plane, thaw mounted onto gelatin-coated microslides, and stored at $-80^{\circ} \mathrm{C}$ until they were reacted.

\section{Antibodies}

Rabbit anti-human tenascin polyclonal antibody was purchased from GIBCO-BRL (formerly from Telios). According to the manufacturer's technical information, the antibody has no cross reactivity with human fibronectin or with other human plasma proteins, but it does have documented species cross reactivity to rat, mouse, pig, and chicken tenascin. We also confirmed the specificity of the antibody using Western blot analysis and ELISA. The antibody was tested against purified human tenascin and fibronectin (both from GIBCO-BRL) across a wide range of dilutions. The antibody recognized the purified tenascin, and no cross reactivity with fibronectin was detected.

This tenascin antibody has also been compared to various monoclonal and other polyclonal antibodies against purified mouse, chicken, or human tenascin (Rettig et al., 1992), and it has been established that it does not cross react with rat laminin, human fibronectin, or human thrombospondin, but it is specific for purified tenascin (Murphy-Ullrich et al., 1991). Tenascin proteins are highly conserved and structurally similar in a wide variety of species, including chicken, rat, mouse, human, cow, and pig (Erickson, 1993; Rettig et al., 1994).

Rabbit anti-human polyclonal laminin antiserum was also purchased from GIBCO-BRL. The antibody has demon- strated cross reactivity to rat laminin but no cross reactivity with human fibronectin or other human plasma proteins in Western blot analysis (GIBCO, technical information). In our laboratory, the antibody has been used in embryonic and postnatal rat tongue and gustatory papillae with results similar to those in sheep.

Mouse monoclonal antibody to GAP-43 from Boehringer Mannheim has known cross reactivity to GAP-43 from rat, cat, hamster, and human regardless of the protein's phosphorylated state (Boehringer, technical information). Mouse monoclonal antibody to HSPG was purchased from Chemicon and has documented cross reactivity with human, bovine, and dog HSPG.

\section{Immunocytochemistry}

Mounted sections were fixed on slides with $4 \%$ paraformal-

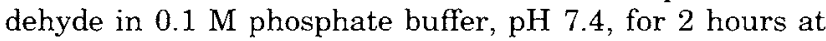
room temperature when immunolocalizing tenascin, HSPG, or GAP-43. Following thorough rinsing in Tris buffer, $\mathrm{pH}$ 7.4 , endogenous peroxidase inhibition was achieved by exposure to $0.05 \%$ hydrogen peroxide in ethanol for tenascin and GAP-43 or in phosphate buffered saline (PBS), $\mathrm{pH}$ 7.4, for HSPG. Sections that were immunoreacted for laminin were fixed and blocked for endogenous peroxidase staining simultaneously by exposure to $0.05 \%$ hydrogen peroxide in $100 \%$ ethanol for 30 minutes at room temperature.

Sections were incubated in a blocking solution that contained $10 \%$ normal goat serum and $0.3 \%$ Triton X-100 (Sigma) in $0.1 \mathrm{M} \mathrm{PBS}, \mathrm{pH} 7.4$, at $4^{\circ} \mathrm{C}$ for $4-15$ hours to eliminate nonspecific binding and to reduce background staining. For HSPG, no Triton X-100 was used in the blocking solution. A carrier solution was made for each immunoreaction with the same contents as the blocking solution but with $1 \%$ normal goat serum instead of $10 \%$. The carrier solution was used to dilute all immune reagents and as a rinse between incubations of the immune reagents.

Tissue was removed from the blocking solution and incubated in the primary antisera: 1) polyclonal rabbit antihuman tenascin (diluted 1:3,000, incubated for 3 hours at $37^{\circ} \mathrm{C}$ );2) polyclonal rabbit anti-human laminin (diluted $1: 300$, incubated for 3 hours at $37^{\circ} \mathrm{C}$ ); 3) monoclonal GAP-43 (diluted 1:2,000, incubated for 48 hours at $4^{\circ} \mathrm{C}$ ); and 4) monoclonal HSPG (diluted 1:100, incubated overnight at $4^{\circ} \mathrm{C}$ ). Controls for each immunoreaction were performed by substituting blocking solution for the primary antisera.

After thorough rinses in the carrier solution, tissue was incubated in the appropriate biotinylated affinity-isolated secondary antibody: goat anti-rabbit immunoglobin, human serum adsorbed, (Kirkegaard and Perry, Inc.), and rabbit anti-mouse immunoglobin (Dako) diluted 1:300 with the carrier for 1 hour at $4^{\circ} \mathrm{C}$.

Tissue was thoroughly rinsed again in the carrier and was incubated in horseradish peroxidase (HRP)-labeled streptavidin (Kirkegaard and Perry, Inc.) in a 1:500 dilution with carrier for 15 minutes at room temperature. The HRP label was visualized with diaminobenzidine (DAB kit; Vector Laboratories) with nickel intensification to produce a black reaction product. Tissue was rinsed thoroughly in Tris buffer, $\mathrm{pH}$ 7.4, dehydrated through ethanols, cleared in xylene, and coverslipped with Permount (Fisher Scientific). 


\section{Analysis}

Sections were viewed with a Leitz diaplan microscope and were traced by drawing tube attachment and/or photographed for analysis across age groups. Fungiform and circumvallate papillae are large complex structures; therefore, a standard papilla region should be analyzed for comparison across developmental groups. We used the middle region of the papilla for analysis, incorporating a complete aspect of the connective tissue core and taste buds in the epithelium.

\section{RESULTS \\ Overview of papilla and taste bud development in fetal and postnatal sheep}

The following brief overview is intended as an orientation only; details are available in reviews (Mistretta, 1991; Mistretta and Hill, 1995) and in later sections of these Results. The mature fungiform papilla in sheep is a large structure $(\sim 300-600 \mu \mathrm{m}$ in diameter) with an epithelial covering over a broad dermal core. Multiple taste buds are located in apical epithelium only. Mature circumvallate papillae can exceed $1.0 \mathrm{~mm}$ in diameter and are composed of a central broad papilla with thinner, flanking nongustatory papilla walls. Taste buds are located in the lateral walls of the central papilla only. Although the precise time of initial light microscopic appearance is not reported, early fungiform and circumvallate papillae are apparent on the fetal sheep tongue at 50 days of gestation. In both early papilla types, epithelial down-growths are obvious, and the broad papilla core readily distinguishes gustatory from surrounding nongustatory filiform papillae. In addition, most fungiform and circumvallate papillae have a single immature taste bud in the papilla apex at this stage. At 80 days of gestation, the papillae are morphologically advanced, with a deeper, wider dermal core and more mature taste buds. Ducts have begun to form for the von Ebner lingual salivary glands that are associated with circumvallate papillae, and taste buds now are distributed both apically and in lateral walls of the circumvallate. Between 100 and 110 days of gestation, taste buds have acquired a pore or surface channel connecting taste bud cells with the oral environment; often more than one taste bud is found apically in fungiform papillae, and the circumvallate papilla walls have begun to "split" and form characteristic trenches. Perinatal papillae are characterized by an essentially mature morphology and numerous taste buds; in fact, taste buds are most numerous in fungiform papillae at this period and decrease in number postnatally (Mistretta et al., 1988). We have organized the Results by presenting data on the early fetal tongue, then data on fungiform papillae, and, finally, data on circumvallate papillae.

The fetal tongue: 35 and 50 days of gestation. At 35 days of gestation (term $=147$ days of gestation), the early fetal tongue has a relatively homogeneous epithelium with occasional undulations but with no obvious fungiform or circumvallate papilla development (Fig. IA). Tenascin immunoreactivity is differentially distributed in the subepithelial connective tissue, is intense in the posterior tongue, and is weaker in the tip (Fig. 1B). Laminin immunoreactivity is distributed in a very thin line under the lingual epithelium, demonstrating an intact basement membrane throughout the tongue epithelium, and is apparent also in the basement membranes of lingual nerves and vessels in the body of the tongue (Fig. $1 \mathrm{C}$ ). The extensive innervation in the early tongue, evidenced with GAP-43 immunoreactivity, has a widespread distribution in the subepithelial connective tissue and throughout the lingual core (Fig. 1D).

By 50 days of gestation, the tongue has increased greatly in size and histologic complexity. Early fungiform papillae, which are not observed at 35 days of gestation (Fig. 1A), are readily apparent now (Fig. 2A) but still are covered with the transient layer of periderm cells. Tenascin immunoreactivity is diffuse throughout the body of the anterior tongue, but it is moderately intense in a band of subepithelial connective tissue and is very intense in a restricted, basement membrane-associated region of the rapidly growing tongue tip (Fig. 2B). The extensive, dispersed lingual innervation and nerves within each fungiform papilla are labeled with GAP-43 immunoreactivity (Fig. 2C).

\section{Fungiform papilla}

$50 D F$. In transverse sections of the anterior tongue, early fungiform papillae are numerous in the epithelium (Fig. 3A). Tenascin immunoreactivity is apparent throughout a band of mesenchyme under the epithelium and is intensely associated with the basement membrane (Fig. $3 \mathrm{~B})$. However, within the connective tissue core of the early fungiform papilla, the tenascin label is either absent, in diffuse patches, or in thin strands at extreme lateral edges of the core (Fig. 3B, inset). On either side of the base of the papilla, tenascin immunoreactivity is intense and is closely associated with the basement membrane.

Laminin immunoreactivity labels the basement membrane of papillae and surrounding lingual epithelium at 50DF (Fig. 3C). In addition to basement membraneassociated label, laminin immunoreactivity is demonstrable within the mesenchyme of the papilla core in a distribution that suggests labeling of neural and/or vascular basement membrane elements (Fig. $3 \mathrm{C}$, inset). However, it is notable that, in papillae with a presumptive taste bud, there is a distinct and large disruption in the immunoreactivity associated with the apical papilla basement membrane (Fig. 3C, inset). These disruptions are under regions of the epithelium where early taste buds form or are forming, and their presence is even suggested in the hematoxylin and eosin section in Figure $3 \mathrm{~A}$ (inset). The disruptions can be up to 40 $\mu \mathrm{m}$ in length and suggest that there might be large discontinuities in the basement membrane itself. Therefore, we examined immunolocalization of a second major basement membrane molecule: HSPG. At 50DF, HSPG immunoreactivity directly parallels the immunolabel for laminin (Fig. 4) and confirms the proposition that the papilla basement membrane has large disruptions under the apical epithelium, where taste buds develop.

GAP-43 immunoreactivity is extremely intense in nerves throughout the tongue and in those that penetrate the connective tissue core of the 50DF fungiform papillae (Fig. $3 D)$. In contrast to the weak tenascin label in the papilla core (Fig. 3B, inset), GAP-43 immunoreactivity seems to fill much of the core (Fig. 3D, inset). There is also some immunoreactivity in the papilla epithelium, where the presumptive taste bud is developing (Fig. 3D, inset). This epithelial immunoreactivity is usually intense but is limited in extent.

$80 D F$ and $100 D F$. The fungiform papilla at $80 \mathrm{DF}$ and 100DF has several layers of epithelial cells over a broad connective tissue core (Fig. 5A). Each papilla generally has a single immature taste bud in the apical epithelium. Cells 

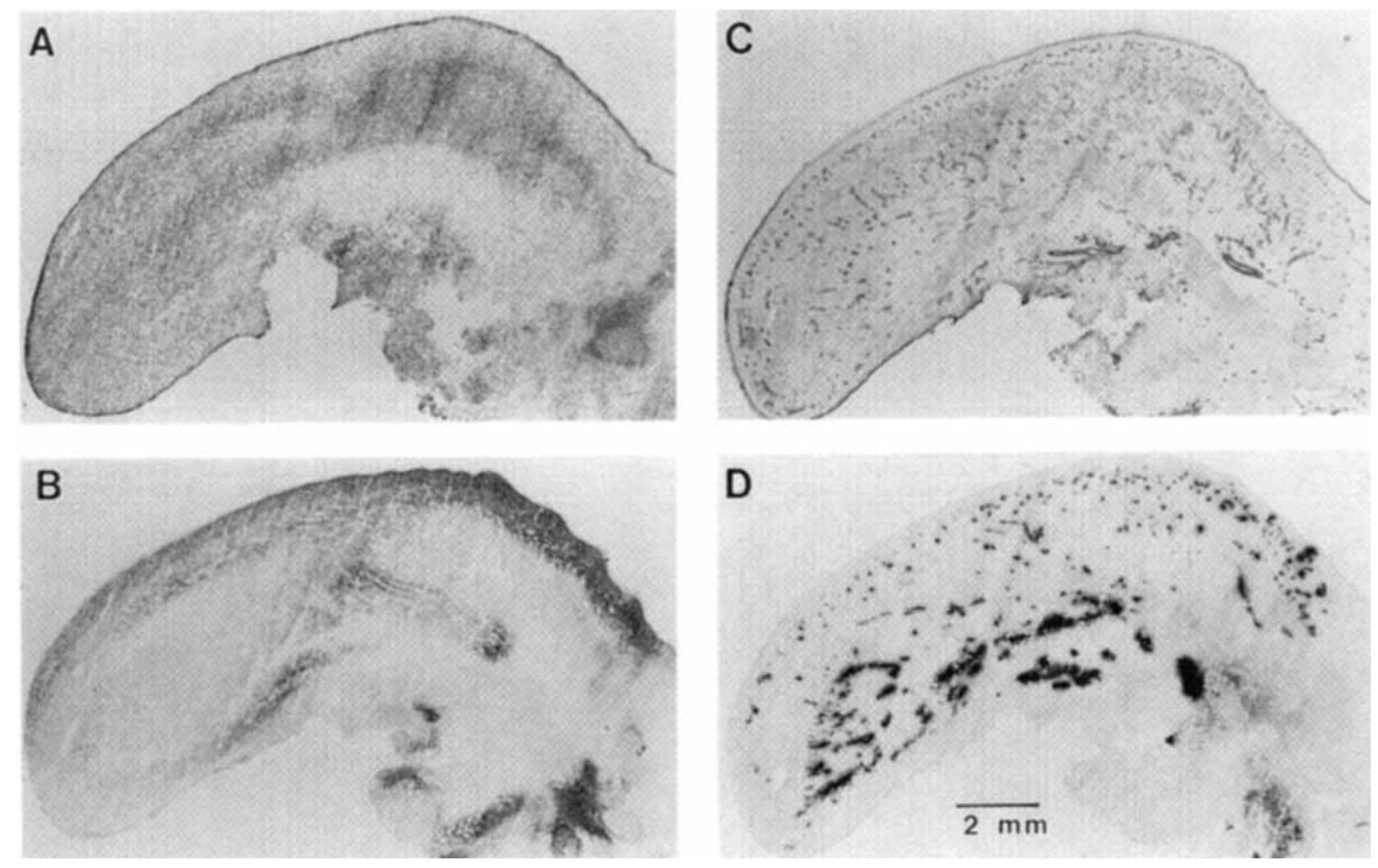

Fig. 1. Photomicrographs of sagittal sections of fetal sheep tongue at 35-40 days of gestation. A: Tongue stained with hematoxylin and eosin illustrates smooth lingual epithelium. B: Tenascin immunoreactivity is intense in a subepithelial band of mesenchyme in the posterior tongue and is moderately intense in the anterior tongue. C: Laminin

of the taste bud are in a large cluster, and not all are oriented longitudinally. Melanocytes and melanin granules are obvious throughout the basal layer of the papilla epithelium and in the taste bud.

A distinct shift in the pattern of tenascin immunoreactivity is seen at $80 \mathrm{DF}$ and $100 \mathrm{DF}$ compared to the fungiform papilla at $50 \mathrm{DF}$. Intense tenascin immunolabel is now observed in the papilla core, but it is highly restricted to the apical one-third of the papilla under lingual epithelium, where taste buds are developing (Fig. 5B). The thick lacework of tenascin immunoreactivity is not seen in adjacent nongustatory, filiform papillae.

The papilla and the contiguous general, lingual, epithelial basement membrane is intensely immunoreactive for laminin (Fig. 5C). Like that seen at 50DF, laminin immunoreactivity is also obvious within the central papilla core in regions where nerves and vessels are located. However, apical discontinuities in the papilla basement membrane are again apparent in the $80 \mathrm{DF}$ and $100 \mathrm{DF}$ papilla, although they are usually more punctate in pattern than those at 50DF. These disruptions are also suggested from hematoxylin and eosin staining (Fig. 5A). The discontinuities are replicated with HSPG immunoreactivity and directly parallel zones of GAP-43 immunoreactivity in the papilla apex (Fig. 6 ).

GAP-43 immunoreactivity is intense in nerve fibers within the papilla core and in the branching fibers under the taste

immunoreactivity labels basement membranes of the lingual epithelium and nerves and vessels throughout the tongue. D: GAP-43 immunoreactivity labels nerve fibers distributed throughout the core of the tongue and under the epithelium.

bud (Fig. 5D). Interestingly, in the apical papilla region, where GAP-43 label demonstrates extensive branching of nerve fibers, tenascin label is most intense (Fig. 5B). In contrast, tenascin is absent in the lower central papilla core, where large bundles traverse the tissue. Examination of serial sections demonstrates that GAP-43 immunoreactivity is also seen within the large, immature taste bud, but it is not located in other cells of the papilla epithelium (Fig. 5D-F).

$110 D F$. At $110 \mathrm{DF}$, the fungiform papillae often have one or two smaller, more laterally located taste buds compared to the single large, central taste bud that is typical at $80 \mathrm{DF}$ and $100 \mathrm{DF}$ (Fig. 7A). Tenascin immunoreactivity remains intense and is restricted to about the apical one-third of the papilla core (Fig. 7B).

Immunoreactivity for laminin and HSPG indicates smaller basement membrane interruptions than at $80 \mathrm{DF}$ through 100DF (Fig. 7C,D), although the nature of the immunolabel in the apical papilla basement membrane is clearly distinctive under taste buds. Also, discontinuities are suggested in the hematoxylin and eosin section of taste buds in Figure 7A. GAP-43 label is located within each of the multiple taste buds now present in the papilla, essentially filling the buds but not located in the surrounding epithelium (Fig. 7E).

$130 D F$, perinatal, and adult. The larger fungiform papillae at $130 \mathrm{DF}$ and perinatal stages often have numerous taste buds (Fig. 8A). Tenascin continues to immunolocalize 

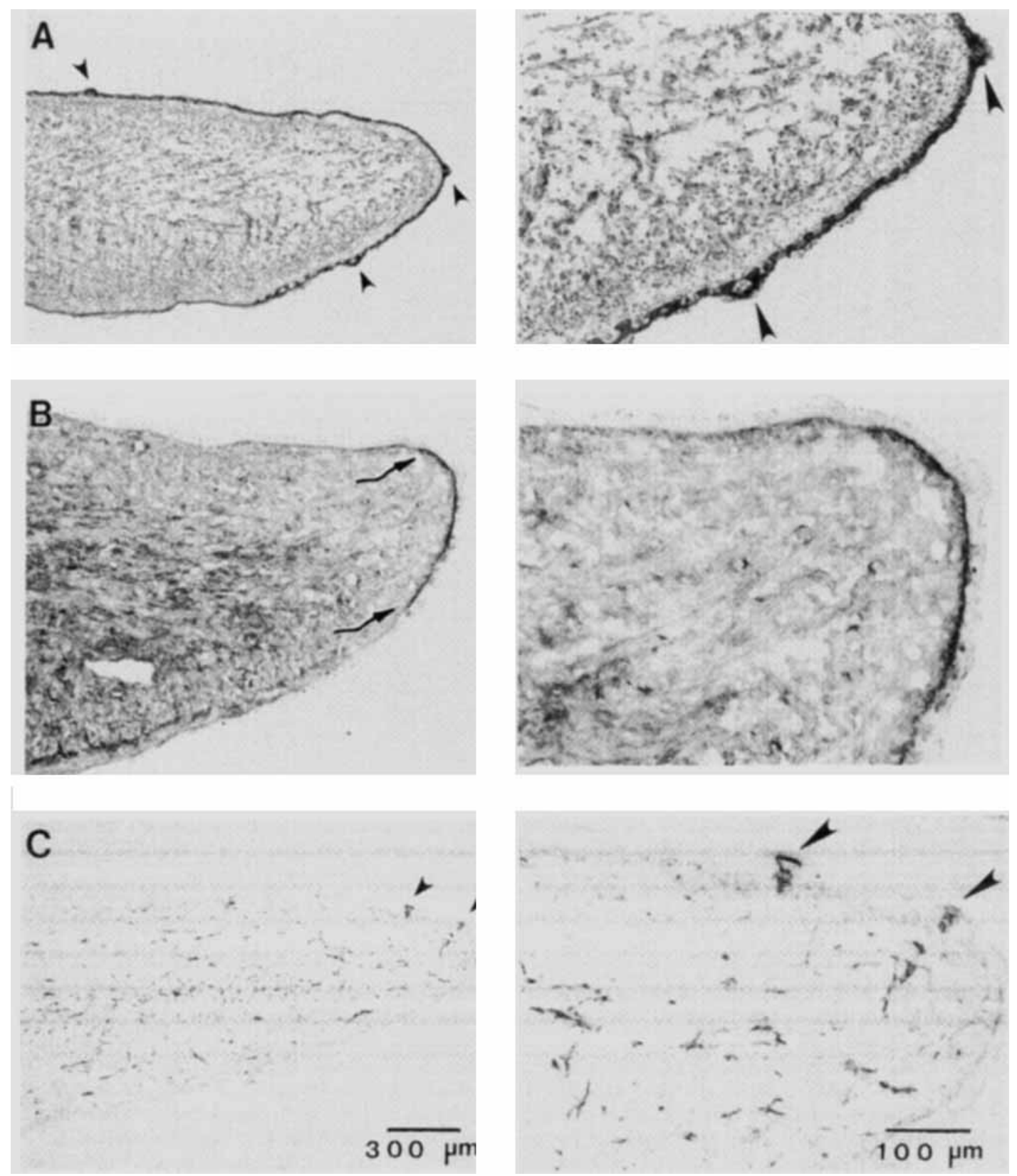

Fig. 2. Photomicrographs of sagittal sections of anterior fetal sheep tongues at 50 days of gestation (low power, left; high power, right). A: Section stained with hematoxylin and eosin illustrates numerous immature fungiform papillae (arrowheads). B: Tenascin immunoreac-

tivity is very intense in a narrow band just under the epithelium of the growing tongue tip (arrows). C: Nerve fibers throughout the tongue are labeled with GAP-43 immunoreactivity, which is intense within the core of the early fungiform papillae (arrowheads).

in the apical one-third of the papilla core, directly under taste bud-bearing regions of papilla epithelium (Fig. 8A). In these more mature fungiform papillae, laminin immunoreactivity within the papilla core directly parallels the innervation labelled with GAP-43 (Fig. 8B,C). Disruptions in laminin immunoreactivity in the papilla basement membrane are no longer apparent.

In the large adult papilla, tenascin immunoreactivity is distributed throughout the upper one-third of the papilla core in a lacework that is intense (Fig. 8D). Immunoreactivity for both laminin and HSPG is intense in the basement

membrane of the adult papilla, nerves, and vessels (not illustrated). Antibody to GAP-43 did not label innervation in the adult fungiform papilla or tongue, although adult tissue was immunoreacted in the same experiments as younger fetal tissue in which a positive reaction was observed and a range of dilutions of the primary antibody and various fixation times were used.

\section{Summary: Fungiform papilla}

In summary, tenascin immunoreactivity shifts during development from a very weak label in the early fungiform 

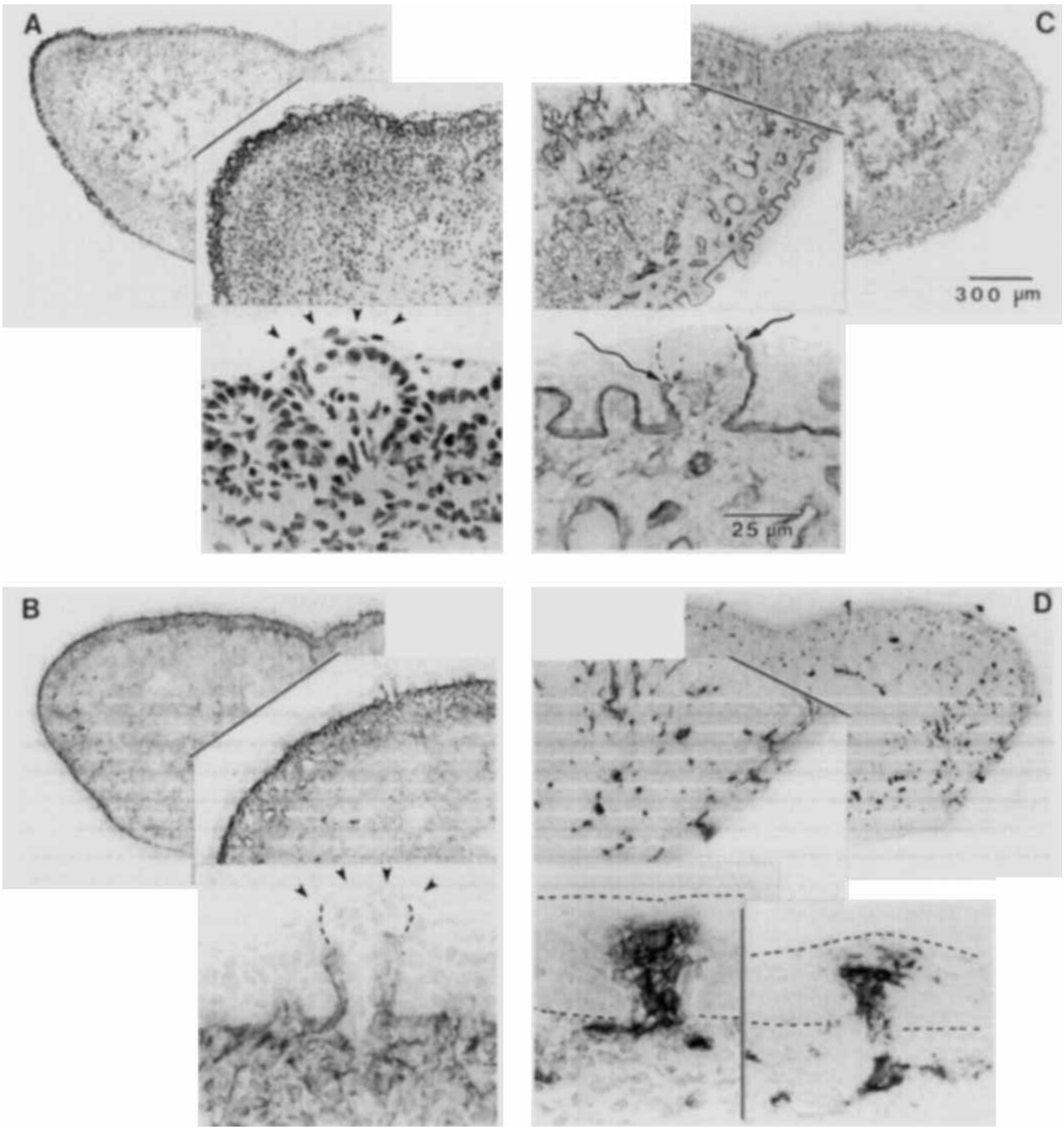

Fig. 3. Transverse sections of fetal sheep tongue at 50 days of gestation and insets of fungiform papillae. For each tissue section, a higher power micrograph of one portion of the tongue illustrates the subepithelial distribution of the immunolabel in detail. A: The early fungiform papillae have a broad connective tissue core and several epithelial cell layers (hematoxylin and eosin). A collection of cells that form the immature taste bud is present in the apical papilla epithelium (arrowheads). B: Tenascin immunoreactivity is distributed in a broad, intense band under the lingual epithelium, is very intensely associated with the epithelial basement membrane, and is less intense throughout the body of the tongue. Tenascin is noticeably absent or weak in the fungiform papilla core (inset), except for occasional thin strands at the lateral areas. The developing taste bud in the apical epithelium is

indicated with arrowheads and dashed lines. C: Laminin immunoreactivity outlines basement membranes of blood vessels and nerves throughout the tongue and is continuous in the lingual and papilla basement membrane. However, in the apical papilla epithelium, there is a large gap in immunoreactivity within the basement membrane under the developing taste bud (dashed lines outline the taste bud, and arrows indicate the extent of basement membrane gap in inset). D: GAP-43 immunoreactivity labels nerves throughout the fetal tongue and almost fills the core of the early fungiform papillae. Restricted label is also seen in the apical papilla epithelium in the region of the developing taste bud (insets illustrate two different papillae). Dashed lines indicate limits of the epithelium in insets. 

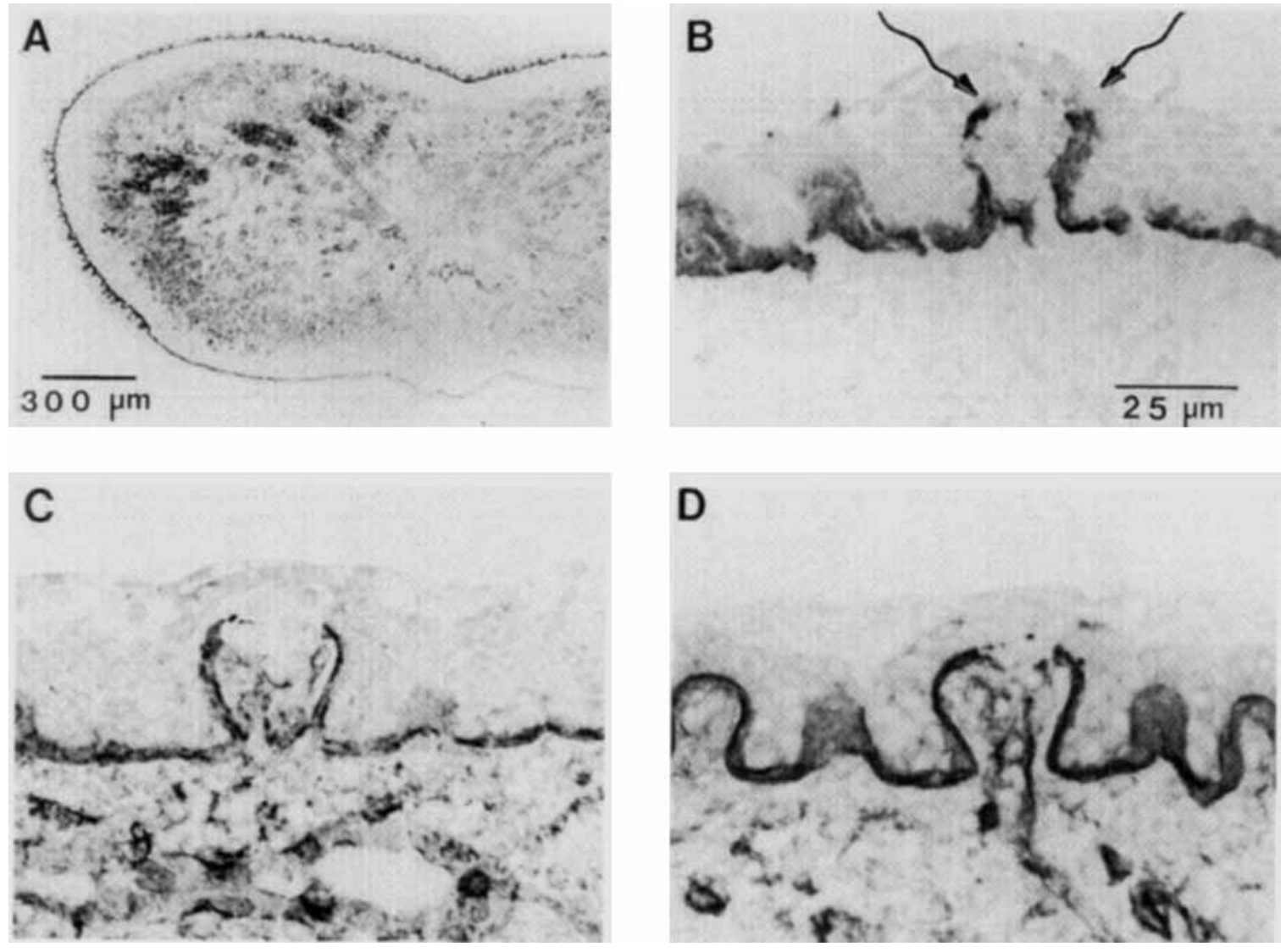

Fig. 4. Transverse sections of fetal sheep tongue and fungiform papillae at 50 days of gestation illustrate disruptions in basement membrane. A,B: Heparan sulfate proteoglycan (HSPG) immunoreactivity throughout the tongue (A) and in a fungiform papilla (B). A large disruption in the immunoreactivity along the basement membrane in

the apical papilla epithelium is apparent in B (arrows). C,D: Examples of basement membrane disruptions in apical epithelium of other papillae indicated by breaks in laminin immunoreactivity. Breaks are under epithelial regions, where taste buds are forming in the papilla epithelium. Scale bar in B also applies to C,D

papilla core to a restricted, intense distribution in the apical papilla core under taste bud-bearing epithelial regions. This distribution persists in the adult papilla. Whereas tenascin immunoreactivity is weak or absent in the core of the early papilla, GAP-43 immunoreactivity densely fills the papilla core. Subsequently, GAP-43 immunoreactivity is intense: It is associated with discrete nerve bundles in the papillae and tongue and also is localized within taste buds. This pattern continues through the perinatal period, but GAP-43 label is absent in adult. Laminin and HSPG immunoreactivity demonstrates large breaks in the apical epithelial basement membrane of fungiform papillae under developing taste buds at $50 \mathrm{DF}$ through $100 \mathrm{DF}$. At later stages, there are punctate basement membrane disruptions in these regions, but the large breaks are no longer apparent.

\section{Circumvallate papilla}

$50 D F$. The early circumvallate papilla at 50DF usually has one immature taste bud in the apical epithelium of the central papilla structure (Fig. 9A). Flanking papilla structures that do not contain taste buds surround the central papilla.

Tenascin immunoreactivity in the posterior tongue is distributed diffusely throughout a band of subepithelial connective tissue (Fig. 9B,C). Within the circumvallate

papilla, both in the central gustatory papilla and in the flanking nongustatory structures, the label is moderately intense and is distributed in threads or in broad patches.

Laminin immunoreactivity is intensely associated with the basement membrane of circumvallate papillae and the surrounding epithelium and, within the papilla core, is distributed in association with nerves and vessels (Fig. 9D). Breaks are apparent in the basement membrane of the apical papilla epithelium in regions of initial taste bud formation and are confirmed with HSPG immunoreactivity (not illustrated). These basement membrane disruptions are essentially similar to those in fungiform papillae at this age and are suggested from hematoxylin and eosin staining in Figure 9A.

GAP-43 immunoreactivity fills much of the central portion of the papilla core (Fig. 9E), as it does in the fungiform papilla at this age, or, in broader papillae, is associated with dispersed nerve bundles (Fig. 9F). The GAP-43 label generally corresponds to tenascin-free regions (compare Fig. $9 \mathrm{E}, \mathrm{F}$ to Fig. 9B,C). GAP-43 immunoreactivity is also located in the apical papilla epithelium, where the initial large, presumptive taste bud develops (Fig. 9E,F).

$80 D F$ and $100 D F$. Tenascin immunoreactivity is distributed throughout the central core of the circumvallate papilla and in the flanking (nongustatory) papilla struc- 

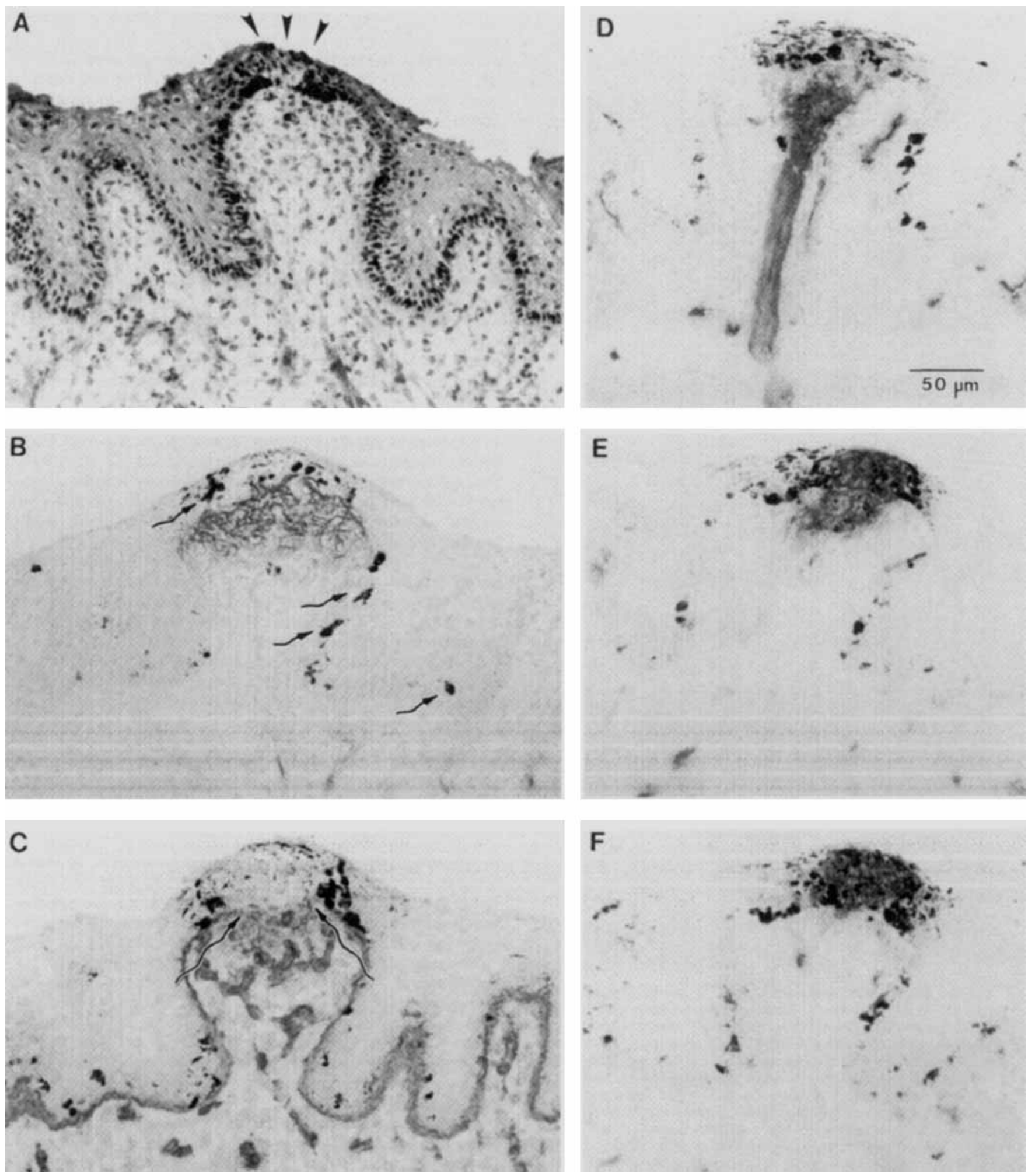

Fig. 5. Photomicrographs of fungiform papillae from fetal tongues at 80 or 100 days of gestation. A: Papilla stained with hematoxylin and eosin illustrates the multilayered papilla epithelium, single immature taste bud (under arrowheads), and large connective tissue core. Melanocytes and melanin granules are present within basal layers of the epithelium and in the taste bud. B: Tenascin immunoreactivity is now highly restricted to the apical connective tissue of the papilla core, under taste bud-bearing epithelial regions. In the nongustatory, filiform papillae on either side of the fungiform, tenascin immunoreactivity is absent. Note that the extremely dark structures in the basal layer of the epithelium and within the developing taste bud are melanocytes

and melanin granules, which are not to be confused with the immunoreactivity; examples are noted with arrows. C: Laminin immunoreactivity is intense and continuous throughout the lingual and papilla basement membrane but is interrupted under the developing taste bud (arrows delimit interruption). Like the photomicrograph in A, melanocytes and melanin granules are obvious in the papilla and the taste bud. D-F: GAP-43 immunoreactivity shown in serial sections labels the nerve bundle within the fungiform papilla core and branches under the apical epithelium and demonstrates intense label within the large, immature taste bud. Scale bar applies to A-F. 

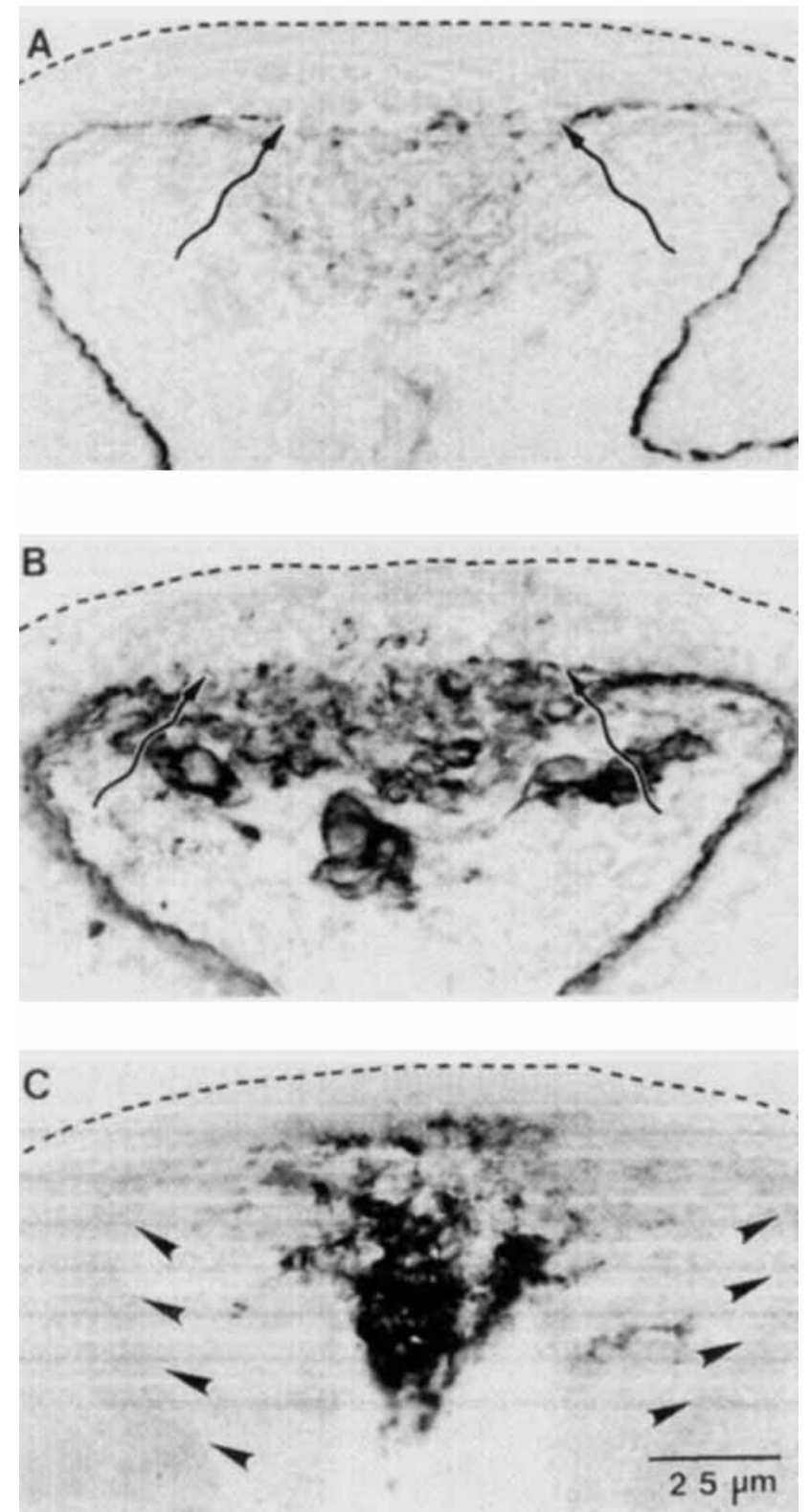

Fig. 6. High-power photomicrographs of nearby sections from a fungiform papilla at 100 days of gestation. Immunoreactivity for HPSG (A) and laminin (B) illustrate the interrupted basement membrane in the apical papilla epithelium. GAP-43 immunoreactivity (C) labels the dense network of branching fibers under the apical papilla epithelium. Each tissue section is thick with dense immunoreactivity. Therefore, in $\mathrm{A}-\mathrm{C}$, each micrograph has been focused on the region where the basement membrane is disrupted (between arrows in $A$ and $B$ ). Dashed lines indicate the outer extent of the papilla epithelium in $\mathrm{A}-\mathrm{C}$, because none of the antibodies reacts with epithelial cells. In $C$, arrowheads indicate the basement membrane of the papilla epithelium, which is not immunoreactive with the GAP-43 antibody for neural elements.

tures at 80 and at 100DF (Fig. 10A). The patchy nature of the immunoreactivity is apparent at higher magnification (Fig. 10B) and contrasts with the more filamentous label at 50DF (Fig. 9). Laminin immunoreactivity distinguishes basement membranes throughout the posterior tongue, including those associated with von Ebner and mucous glands (Fig. 10C). Large disruptions in the basement membrane of the papilla epithelium are not apparent at this age (Fig. 10D): However, punctate disruptions are sometimes observed. Furthermore, the quality of the immunoreaction in the epithelial basement membrane of the central taste bud-bearing papilla and along the clefts of von Ebner's glands is less delineated and continuous than in flanking structures, where a thick, intense, and continuous line of immunoproduct is observed (Fig. 10D,E).

II0DF. By 110DF the circumvallate papilla has a more mature morphology, and flanking papilla structures have split away from the central gustatory papilla (Fig. 11). Tenascin immunoreactivity at this stage has shifted to an intense distribution around the central papilla perimeter (Fig. 11A,B), with weaker patches throughout the central papilla core. Interestingly, label for tenascin is also seen along the basement membrane at the base of the papilla, where ducts of the von Ebner salivary glands are forming. Although some immunoreactivity remains in the nongustatory flanking papillae, this is now very weak relative to that in the central papilla and, where present, is in a very thin band. Intense laminin immunoreactivity is observed throughout basement membranes of posterior tongue structures (Fig. 11C) and labels large nerve bundles within the central papilla that branch to distribute to taste budbearing epithelial zones (Fig. 11C,D).

GAP-43 immunoreactivity confirms the distribution of nerve bundles within the center of the papilla core and branches toward taste bud-bearing portions of the epithelium (Fig. 11E,F). The large central nerve bundle runs in a tenascin-free region (compare Fig. $11 \mathrm{~A}, \mathrm{~B}$ to Fig. $11 \mathrm{E}, \mathrm{F}$ ). GAP-43 immunoreactivity is also located within each of the circumvallate taste buds but is not found in surrounding papilla epithelium (Fig. $11 \mathrm{~F}$ ); this is directly comparable to the fungiform papilla at this age.

$130 D F$, perinatal, and adult. The very large circumvallate papilla at $130 \mathrm{DF}$ and at older ages has numerous taste buds, and some of these are still located in the apical papilla epithelium (Fig. 12A,B). Tenascin immunoreactivity at $130 \mathrm{DF}$ and perinatal stages is now very restricted around the perimeter of the connective tissue core of the central papilla (Fig. 12C). This contrasts sharply with the flanking nongustatory papillae, where only a thin band of apical immunoreactivity is sometimes observed. The nature of the immunoreaction in connective tissue under taste budbearing epithelium is illustrated in Figure 12D.

The extensive central papilla innervation is indicated by laminin and HSPG immunoreactivity within basement membrane structures associated with the nerve bundles (Fig. 12E-H). Like those seen in younger papillae, the large nerve bundles in the base and center of the papilla travel in a tenascin-free zone. Differential staining of glandular tissue in the posterior tongue is apparent with laminin and HSPG reactions.

In the adult circumvallate papilla, tenascin immunoreactivity persists in a restricted band around the perimeter of the central core and in a much thinner band in the flanking papillae (Fig. 13A). Immunoreactivity for laminin is intense in basement membranes of the papilla epithelium, nerves, and vessels (Fig. 13B).

\section{Summary: Circumvallate papilla}

Tenascin immunoreactivity alters from a thread-like, diffuse distribution in the early circumvallate papilla to a highly restricted, intense pattern around the perimeter of the papilla core in later stages. The restricted pattern 

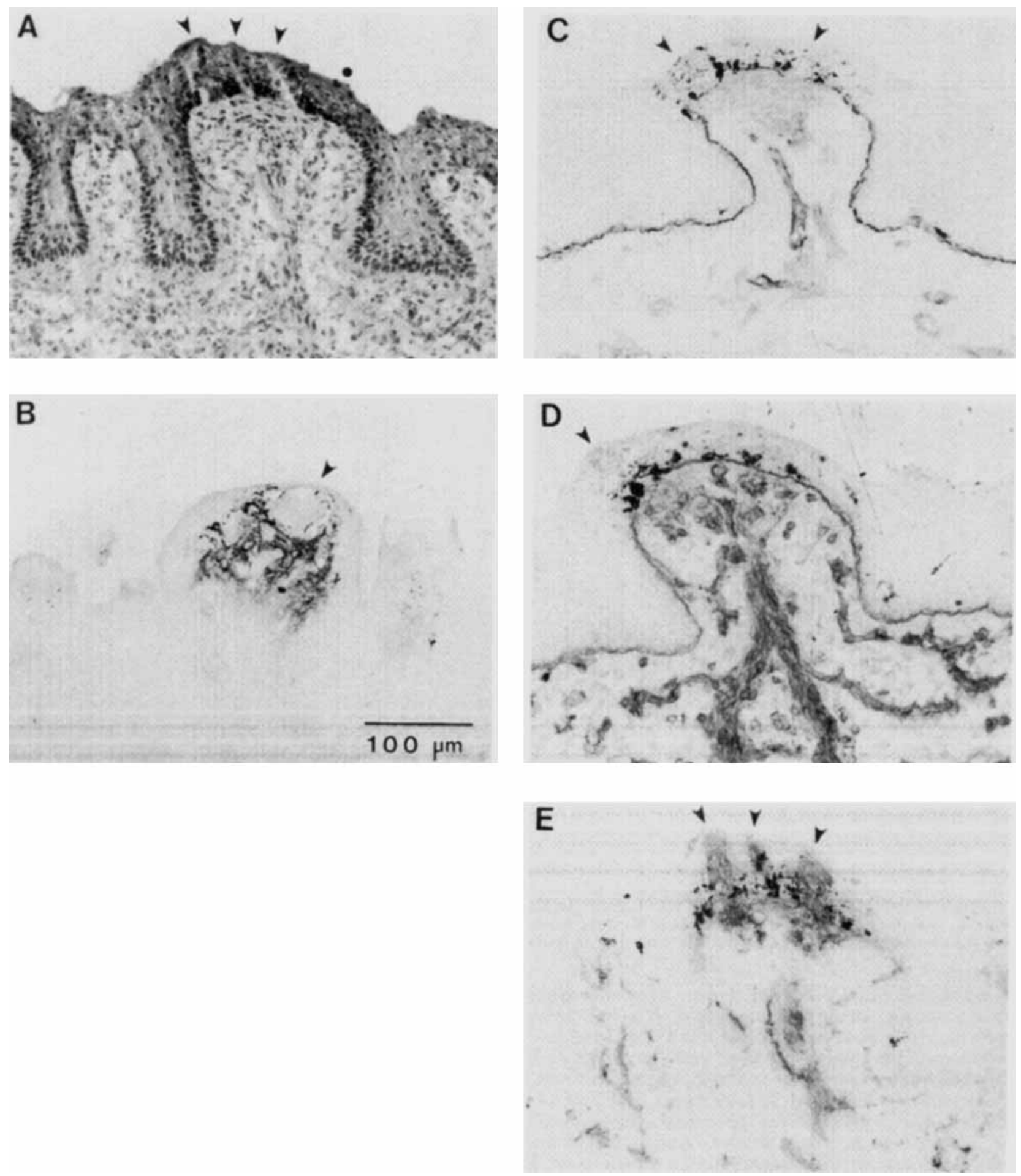

Fig. 7. Photomicrographs of fungiform papillae at 110 days of gestation. Sections photographed in $\mathrm{A}$ and $\mathrm{E}$ are contiguous. A: Fungiform papillae at this age often have more than one taste bud in the apical epithelium, as illustrated with hematoxylin and eosin staining. Three taste buds are marked with arrowheads, and a fourth taste bud (dot) appears in other sections. B: Tenascin immunoreactivity remains restricted to the apical portion of the fungiform papilla core. A

single large taste bud is apparent in the epithelium (arrowhead). C,D: HPSG (C) and laminin (D) immunoreactivity demonstrates that disruptions persist in the basement membrane under developing taste buds (arrowheads). E: GAP-43 immunoreactivity is no longer diffuse but is distinctly associated with lingual nerves and fibers in the fungiform papilla core. Also, the immunolabel is intense within each of the developing taste buds (arrowheads). Scale bar applies to A-E. 

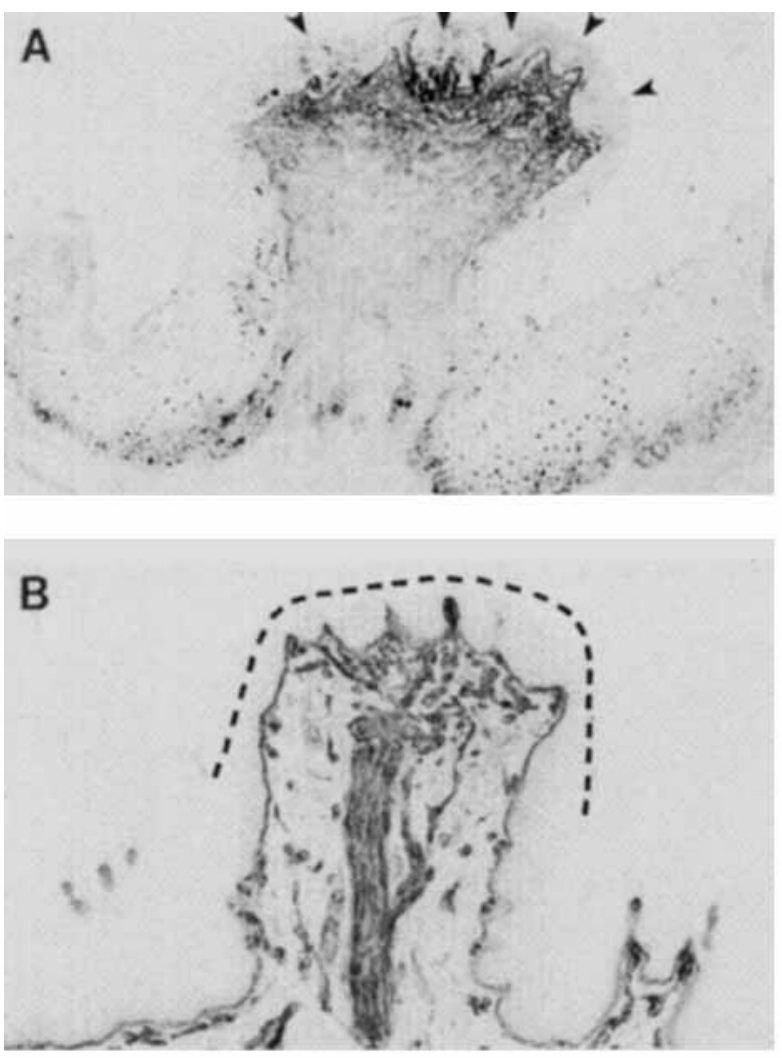

Fig. 8. Photomicrographs of fungiform papillae at 130 days of gestation to the perinatal period $(\mathrm{A}-\mathrm{C})$ and in adult (D). A: The large, mature fungiform papilla usually contains numerous taste buds (arrowheads). Tenascin immunoreactivity is intense in the apical portion of the papilla core under the taste buds. B: Laminin immunoreactivity labels the papilla innervation and epithelial basement membrane. A dashed line outlines the outer epithelial limit on the fungiform papilla. C: GAP-43 immunoreactivity labels nerve fibers in the large bundle within the central papilla core and smaller branches that innervate

persists in the adult. In addition, there is a loss of tenascin immunoreactivity from the nongustatory flanking papilla structures in the later stages. Laminin and HSPG immunoreactivity indicates basement membrane breaks in early circumvallate papillae similar to those in fungiform papillae: Disruptions are punctate at later stages. Like that seen in the fungiform papilla, GAP-43 immunoreactivity fills the early papilla core but only defines nerves bundles and branches thereafter. Progressing from an early, limited label in the region of presumptive taste buds, by $110 \mathrm{DF}$, GAP-43 label is extensive in each of the multiple taste buds but is absent from surrounding epithelial cells.

\section{DISCUSSION \\ Tenascin distributes under taste bud-bearing epithelium}

Within the developing sheep tongue and gustatory papillae, immunolocalization of the extracellular matrix mol-
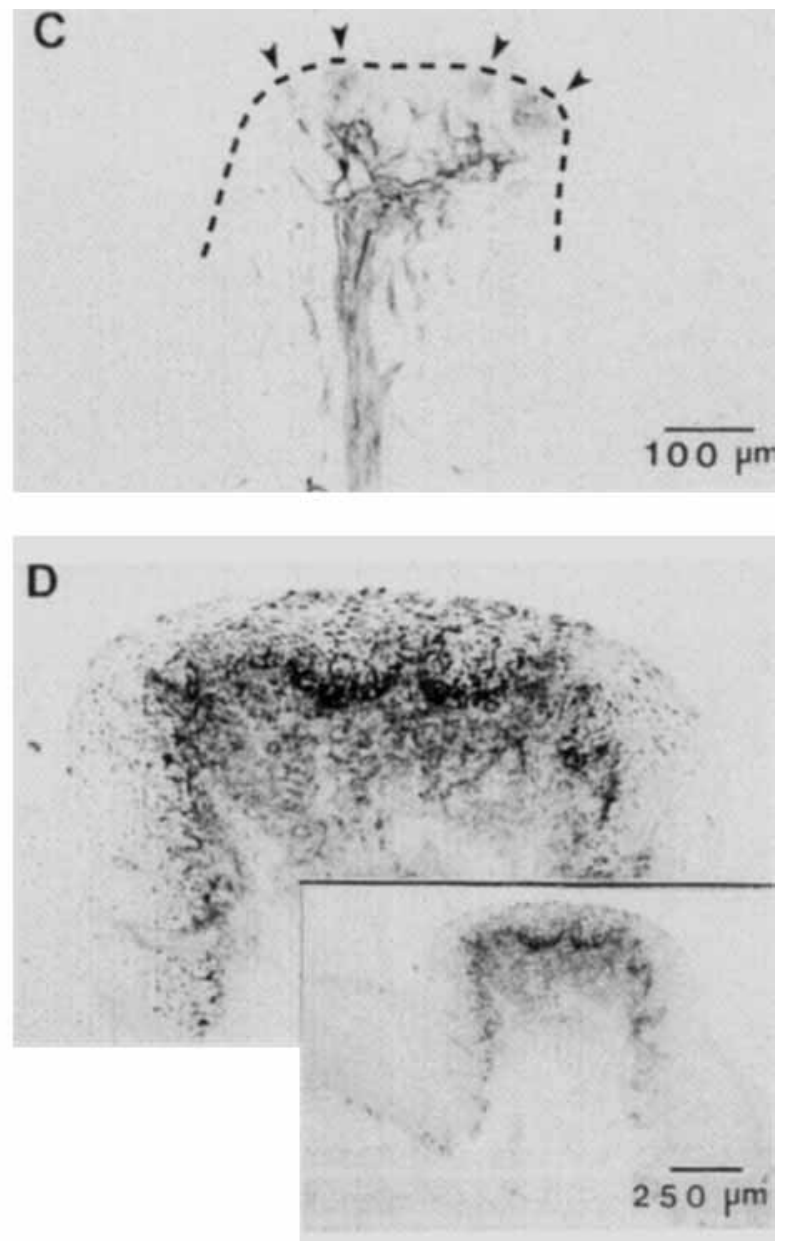

taste buds. A dashed line delimits the outer papilla epithelium. This section is near that illustrated in $B$, and the close match in nerve fiber labeling is apparent. GAP-43 label remains in taste buds (arrowheads) in the perinatal papilla. D: Tenascin immunoreactivity persists in the adult fungiform papilla in the apical core but, as shown in the low-power inset, is absent in other subepithelial locations. Note that the intensely dark structures in the papilla epithelium, especially at the base of the taste buds, are melanocytes and their products. Scale bar in $\mathrm{C}$ also applies to $\mathrm{A}, \mathrm{B}$

ecules tenascin and laminin alters in a manner that reflects morphogenesis in the peripheral taste system rather than histological boundaries. A preliminary report on immunohistochemical distribution of tenascin in developing mouse tongue supports a role for this molecule in rodent papilla morphogenesis as well (Morris-Wiman and Brinkley, 1994). In the early sheep fungiform papilla, tenascin immunoreactivity is very weak within the connective tissue core, but, later, it has an intense and highly restricted distribution within the apical portion of the core. In the early circumvallate papilla on posterior tongue, tenascin immunoreactivity is distributed in filamentous threads in the central papilla core and in diffuse patches in flanking nongustatory structures. However, by $110 \mathrm{DF}$, the immunolabel is restricted to an intense distribution around the perimeter of the core of the central (taste bud-bearing) papilla only. Thus, by about $100 \mathrm{DF}$ in fungiform and $110 \mathrm{DF}$ in circumvallate papillae, tenascin immunoreactivity is restricted to dermal tissues under epithelium where there are taste buds. 

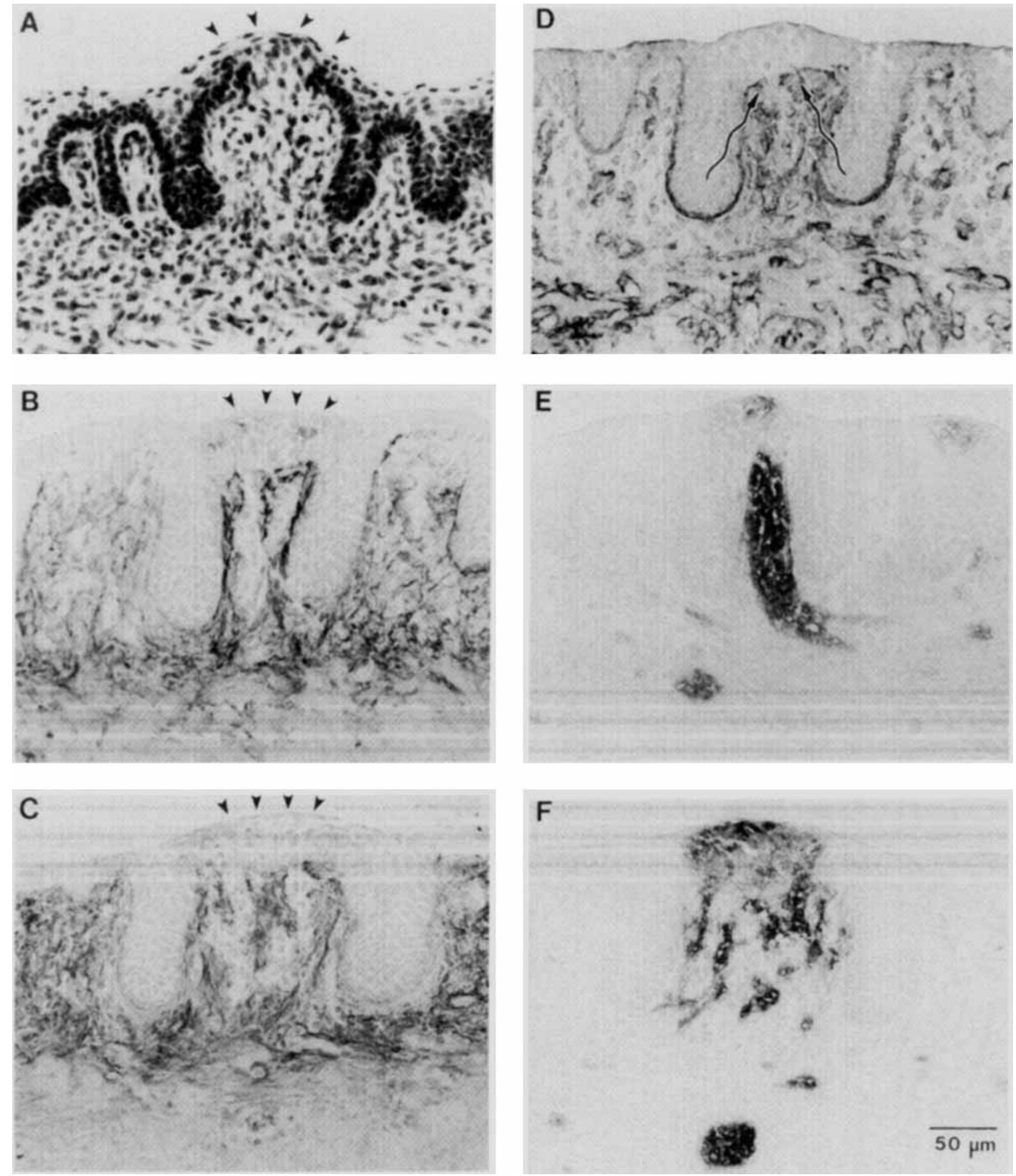

Fig. 9. Transverse sections of circumvallate papillae on the posterior tongue at 50 days of gestation. A: Section stained with hematoxylin and eosin illustrates the broad central papilla with a single immature taste bud in the apical epithelium (arrowheads) and the surrounding, nongustatory papillae that form a wall around the central structure. B,C: Tenascin immunoreactivity in a thin (B) and a broad (C) papilla is distributed in a band within the mesenchyme under the epithelium and is in thin filaments or patches within central and flanking papilla structures. In the central structures of both papillae, a developing taste bud is found (arrowheads). D: Laminin immunoreactivity is associated with the basement membrane of lingual and papilla epithelia and of

nerves and vessels within the papilla and posterior tongue. A disruption is apparent in immunoreactivity of the apical papilla basement membrane (arrows) just under the immature taste bud. E,F: GAP-43 immunoreactivity in nearby sections of the same papillae photographed for tenascin in B and C. Within the thinner papilla (E), GAP-43 label virtually fills the central papilla core: Within the broad papilla $(F)$, the label is in large patches associated with nerve bundles. GAP-43 immunoreactivity is also observed within the immature taste bud of each papilla. The GAP-43 label generally corresponds to a tenascin-free zone (compare to $\mathrm{B}$ and $\mathrm{C}$ ). Scale bar applies to $\mathrm{A}-\mathrm{F}$. 

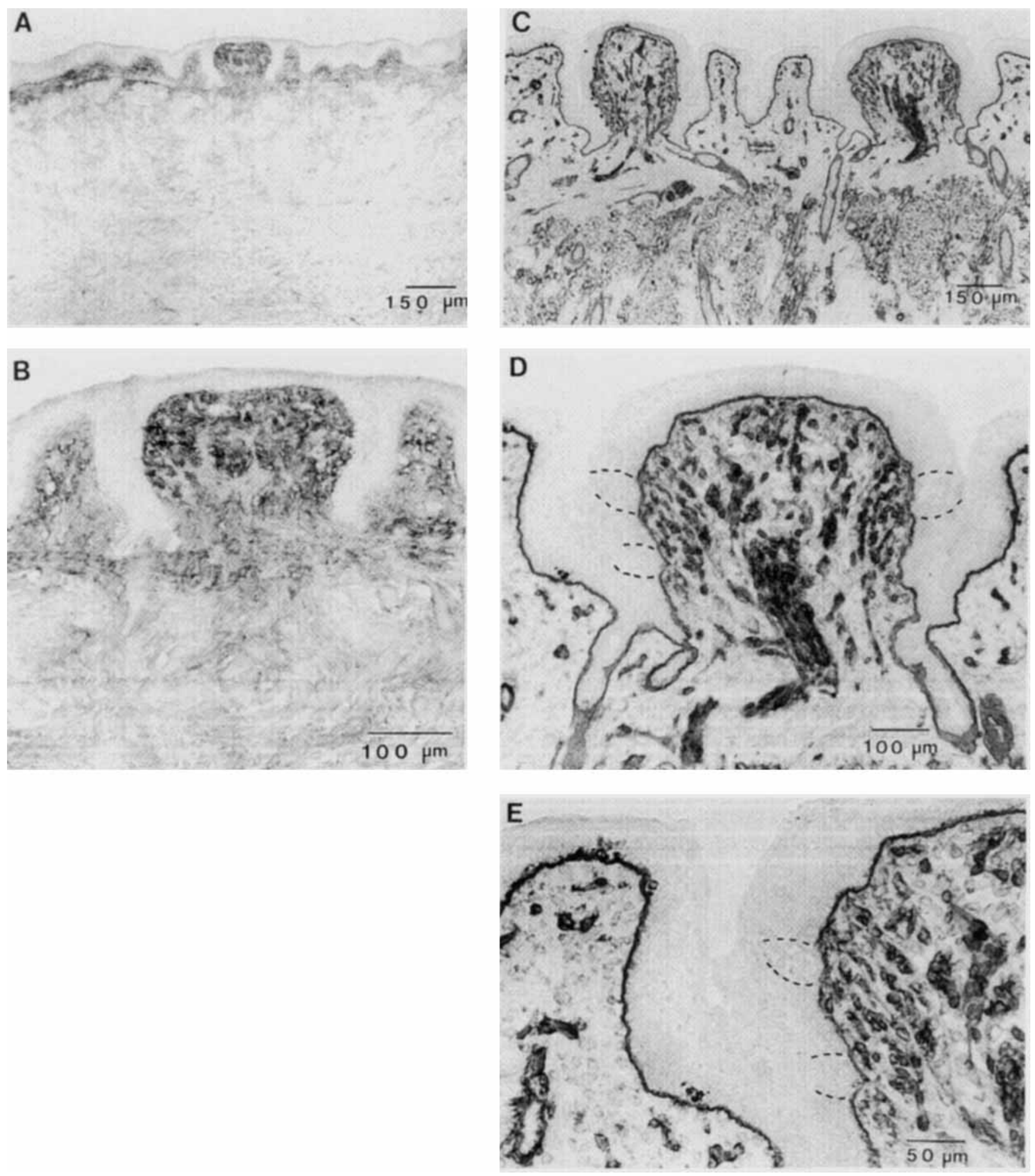

Fig. 10. Transverse sections of fetal posterior tongue and circumvallate papillae at 80 and 100 days of gestation. A,B: Tenascin immunoreactivity remains in a subepithelial band of connective tissue and is in broad patches throughout central and flanking papilla structures. At higher magnification $(B)$, it is apparent that the immunolabel is less filamentous than at 49-51 days of gestation. C-E: Laminin immunoreactivity is present throughout epithelial, neural, vascular, and glandu-

lar basement membranes of two circumvallate papillae and flanking structures $(\mathrm{C})$. Within the central gustatory papilla, there are no obvious basement membrane disruptions; three taste buds are marked with dashed lines (D). However, the nature of the immunolabel along the central papilla basement membrane is less delineated than in the flanking papilla. Two taste buds in the central papilla are noted with dashed lines $(\mathrm{E})$ 

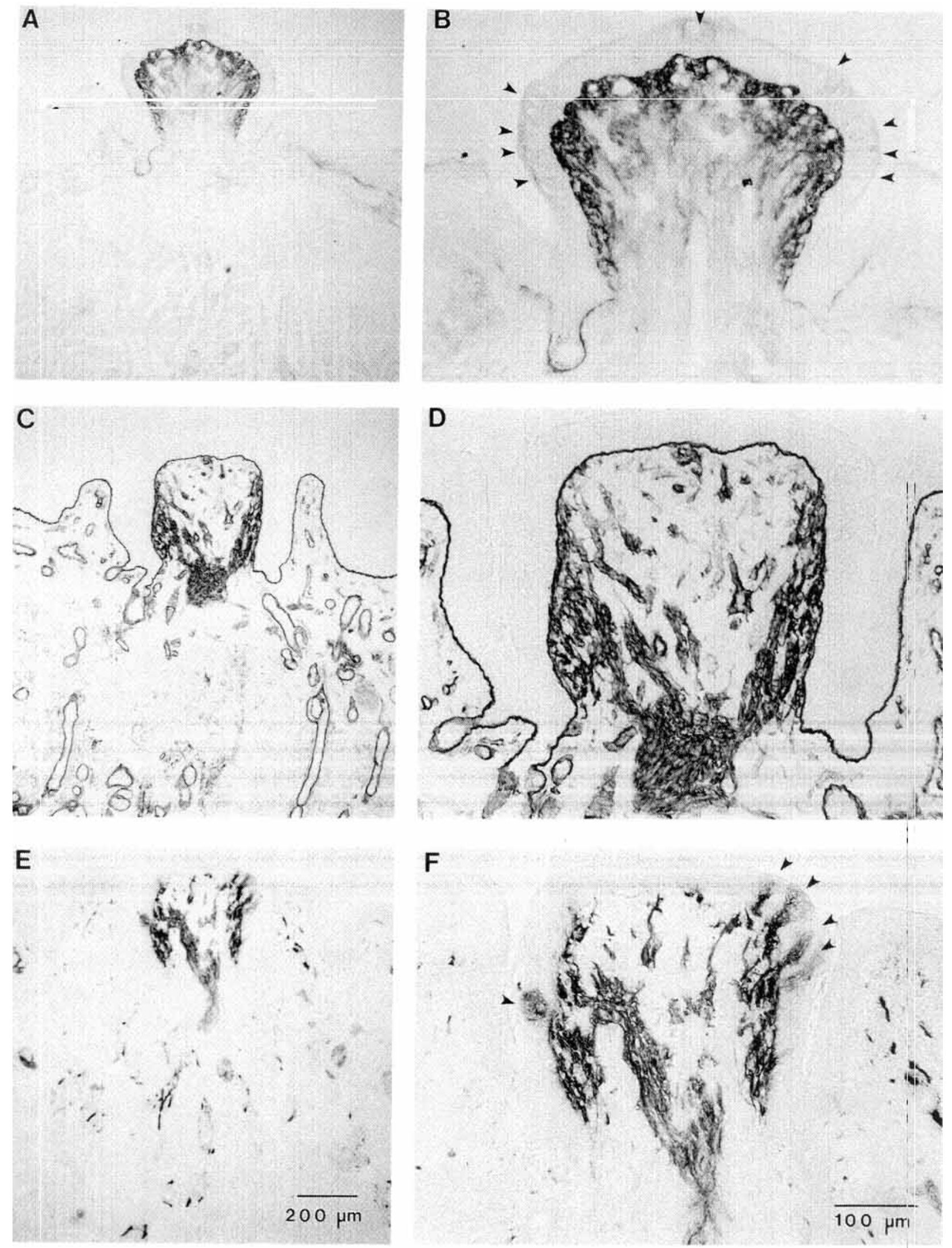

$\mathbf{F}$

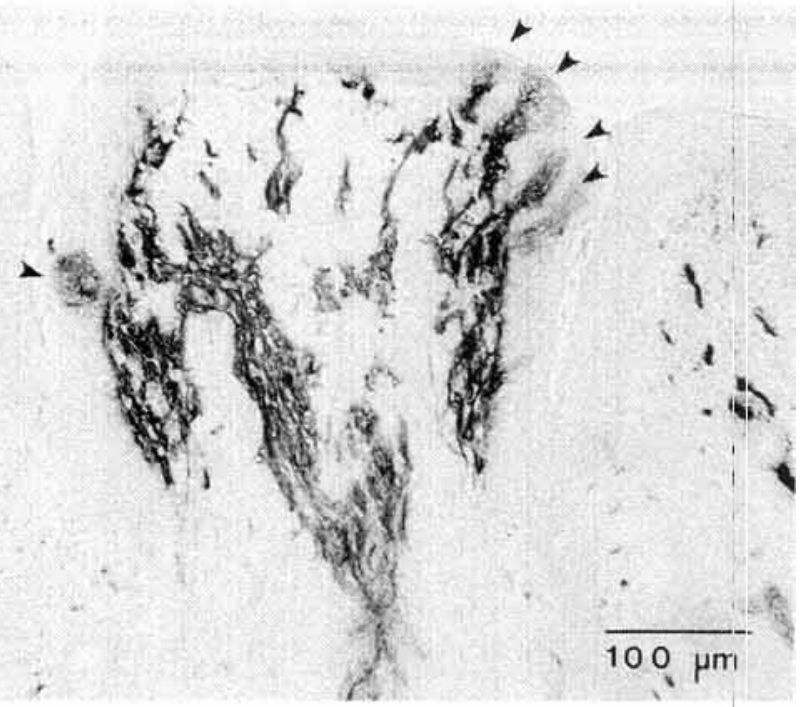

Fig. 11. Circumvallate papillae at 110 days of gestation. By this stage, the flanking papilla structures have split from the central gustatory papilla to form an encircling trench or moat. A,B: Tenascin immunoreactivity is now highly restricted in a connective tissue band around the perimeter of the central papilla only. Taste buds are indicated by a small arrowhead over the respective epithelium. Within the flanking nongustatory papillae, temascin label is absent or weak. C,D: Laminin immunoreactivity is continuous and intense along the

epithelial basement membrane and strongly labels the large nerve bundle and branches within the central papilla. E,F: GAP-43 immunoreactivity demonstrates the large central nerve bundle entering the papilla base and numerous branches around the papilla perimeter as nerves approach taste buds (arrowheads) within the epithelium. GAP-43 label is also distributed within each of the taste buds. The large nerve bundle at the papilla base travels in a tenascin-free region (compare to B). Scale bar in $E$ also applies to A,C; F also applies to B,D. 

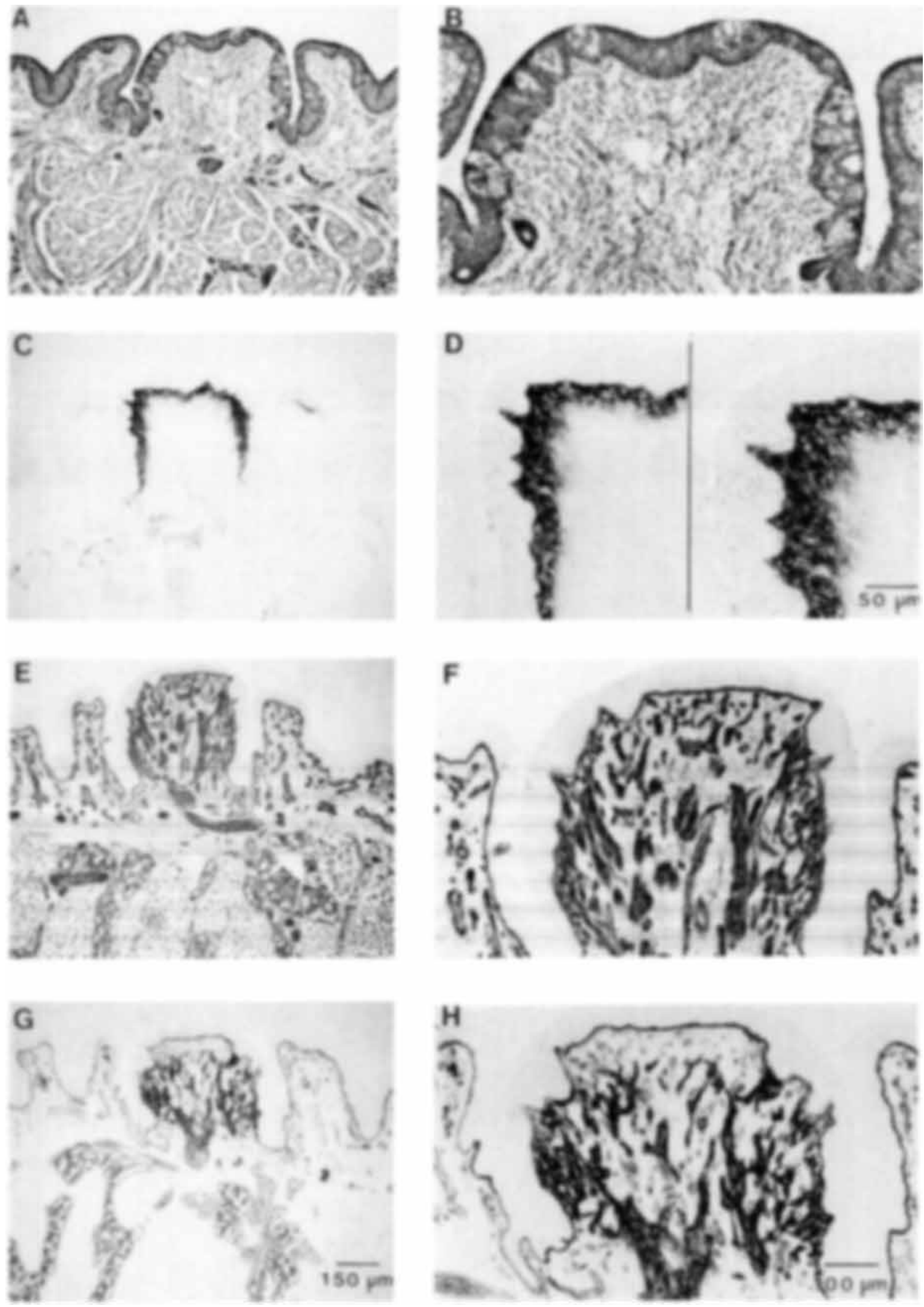

Fig. 12. Circumvallate papillae at 130 days of gestation and the perinatal period. A,B: Section stained with hematoxylin and eosin to illustrate the large central papilla with numerous taste buds in apical and lateral papilla epithelium and the broad flanking papilla structures. C,D: Tenascin immunoreactivity is very intense in a restricted zone around the perimeter of the central papilla core. The immunopositive band alters abruptly at a short distance from the perimeter ( $D$ and inset). Numerous taste buds are present in the central papilla epithelium. In flanking papillae, an extremely thin band of immunoreactivity

is confined under the apical epithelium. E,F: Laminin immunoreactivity is continuous throughout the basement membrane of posterior tongue and papilla epithelia, nerves, and acinar cells and ducts of von Ebner's glands. G,H: HPSG immunoreactivity mirrors label for laminin but is absent from acinar cells of von Ebner's glands. Laminin and HSPG immunolabel illustrates the large nerve bundle at the papilla base and large branches within the papilla core, all in tenascin-free regions. Scale bar in $\mathrm{G}$ also applies to A,C,E; $\mathrm{H}$ also applies to $\mathrm{B}, \mathrm{D}, \mathrm{F}$. 

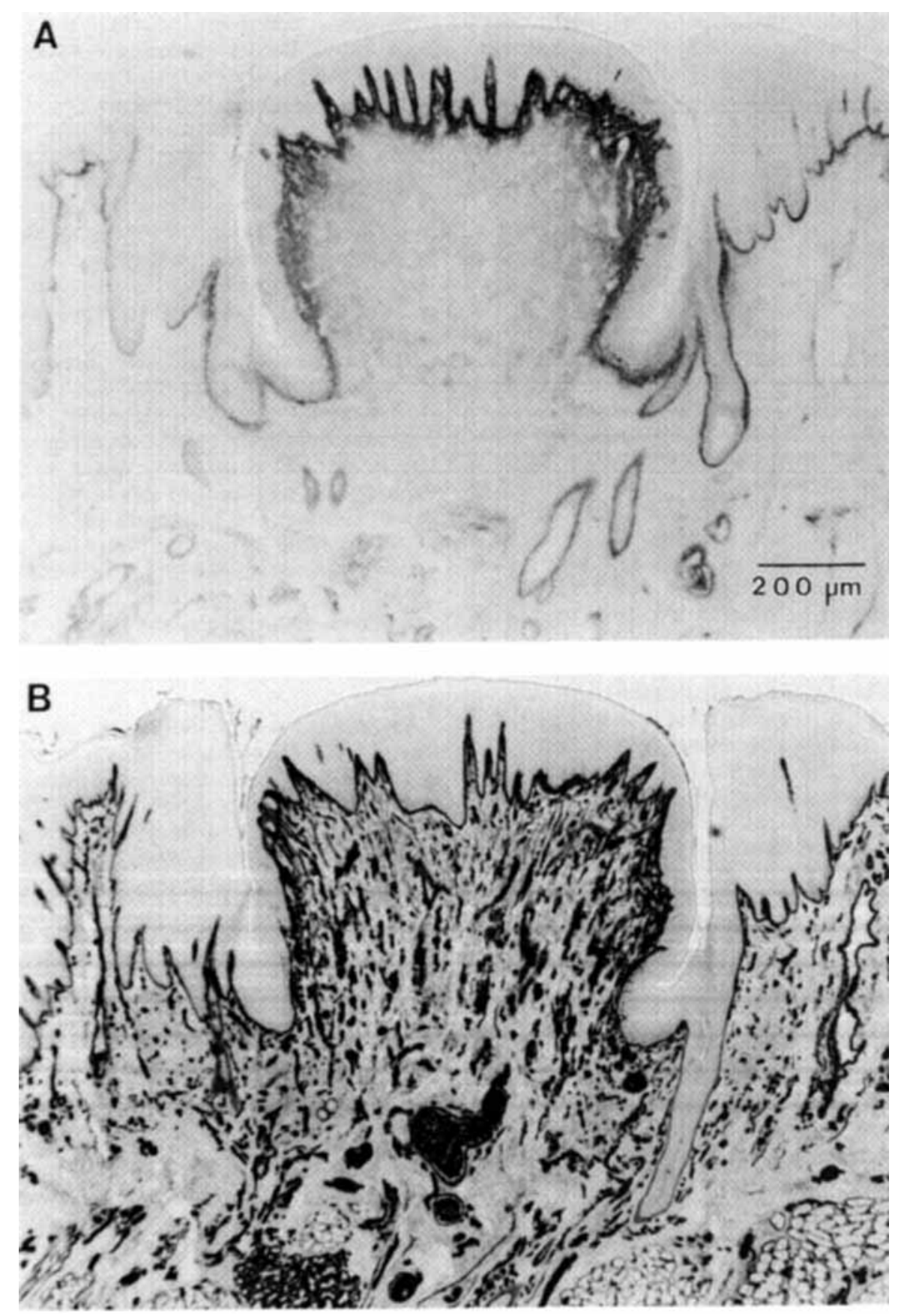

Fig. 13. Circumvallate papillae from adult sheep tongue. A: Tenascin immunoreactivity persists in the adult in an intense band around the perimeter of the central papilla core. Within the large flanking papillae, tenascin label is in a much thinner band around the core. B: Laminin immunoreactivity labels basement membranes of numerous neural, vascular, and glandular elements in posterior tongue.
The shift in tenascin immunolocalization to connective tissue directly under taste bud-bearing epithelium is associated with a key phase in development of the taste system: the period of taste bud maturation and multiplication. We propose that tenascin functions as a deadhesion molecule in contributing to a tissue environment that allows taste bud cells to divide, locomote, and reaggregate and to assume a mature polarity during the differentiation of taste bud cells and during the formation, innervation, and multiplication of the taste buds. Tenascin may well persist in adult papillae under epithelium that contains taste buds, because taste bud cells turn over and are replaced throughout adult life (Beidler and Smallman, 1965).

In other developing organ systems, tenascin apparently functions in cell deadhesion during morphogenesis (ChiquetEhrismann et al., 1991; Murphy-Ullrich et al., 1991). For example, in embryo implantation, tenascin may promote penetration of the uterine wall by disrupting epithelial cell 
adhesion to the basement membrane (Julian et al., 1994). Apparently, tenascin can also generally diminish epithelial cell and fibroblast adhesion to components of the extracellular matrix (Tremble et al., 1994). In organs that acquire a branching pattern during morphogenesis, such as lung (Young et al., 1994), kidney (Aufderheide et al, 1987), and hair follicle (Kaplan and Holbrook, 1994), tenascin is implicated in adhesion regulation during development.

In addition to the thick lacework of tenascin immunoreactivity under epithelium that contains taste buds, tenascin is also apparent along the basement membrane in the apical core of some filiform (nongustatory) papillae and in the numerous secondary papillae at the apex of adult circumvallate papillae under epithelium that does not bear taste buds. This immunoreactivity is distinguished from that under gustatory epithelium by its much thinner band of distribution (see Figs. 11, 12). It is likely that tenascin has different functions in varying locations and during different stages of development in the peripheral taste system. The tenascin immunoreactivity in the apical core of filiform papillae and of the adult circumvallate may relate to a general function for the molecule in maintaining papilla form via interactions with proteoglycans in the extracellular matrix comparable to its function in dermal papillae in the adult skin (Lightner et al., 1989). Differing functions presumably derive from the several variants of the complex tenascin molecule. These variants are generated by alternative mRNA splicing of subunits that are composed of structural domains including epidermal growth factor-like repeats, fibronectin type III repeats, and a terminal region homologous to fibrinogen (Spring et al., 1989; Tucker et al., 1994).

Tenascin also has a demonstrated role in neurite outgrowth and fasciculation in peripheral nerves of the developing chick limb (Wehrle and Chiquet, 1990). A comparable function in the developing taste system is discussed in the later section on GAP-43 immunoreactivity (see below).

In recent experiments, numerous tissues in mice that lacked a functional tenascin gene were examined and were found to be normal (Saga et al., 1992). Several arguments have been advanced to account for the apparent normal histology in these animals (Erickson, 1993; Julian et al., 1994; Tremble et al., 1994): These include the possibility that other genes are activated to produce tenascin-like molecules, which compensate for the tenascin deficiency, and that examination of other tissues or functions at a finer level will reveal abnormalities. The distribution of tenascin in restricted spatial and temporal patterns that relate to morphogenesis of several organs continues to indicate the biological relevance of this molecule in intact animals.

\section{Laminin and basement membrane disruptions during papilla development}

During the period when the first taste buds form in the apical epithelium of fungiform and circumvallate papillae $(\sim 50 \mathrm{DF})$, large disruptions in laminin immunoreactivity are observed in the basement membrane just under developing taste buds. The fact that the breaks in immunoreactivity indeed represent basement membrane disruptions is confrrmed with immunoreactivity for HSPG, a second major molecule of basement membranes (Yurchenco and Schittny, 1990). At later stages of taste bud development and multiplication (80DF through 110DF), the disruptions remain but are generally smaller and more punctate in character.
Basement membrane breaks in the apical papilla epithelium would disrupt adhesion of differentiating taste bud cells to the papilla basement membrane. This disruption could permit cell movement during the process of initial taste bud formation. Laminin can inhibit keratinocyte motility in vitro (Woodley et al., 1988), and it participates in maintenance of the differentiated state of epithelial cells (Beck et al., 1990) and in acquisition and maintenance of cell polarity (Ekblom, 1989). Any of these processes could be potentially counterproductive during establishment of the immature taste bud, when an initially large collection of cells without a specific orientation must aggregate (Mistretta, 1991).

Discontinuities in basement membranes have a role in other developing organs (Yurchenco and Schittny, 1990). For example, during angiogenesis, basement membrane degradation is a key event that exposes endothelial cells to the interstitial connective tissue as a requirement for forming new capillaries (Form et al., 1986). Also, at sites of tumor invasion, loss of laminin and collagen IV in disrupted basement membranes and increased dermal expression of tenascin are observed and are thought to promote cell migration (Van Duinen et al., 1994). The basement membrane breaks in gustatory papilla epithelium could be affected by metalloproteases, which have an active role in basement membrane degradation in other tissues (Chen, 1992).

Clearly, the cells of developing taste buds that lie over a disrupted basement membrane face a very different set of neighboring extracellular matrix molecules than cells over an intact basement membrane zone. The early, large basement membrane breaks would allow direct cell and/or molecular exchange between epithelial and mesenchymal tissue compartments in the developing apical papilla and would also permit extensive access of papilla innervation to developing taste buds. The breaks could also serve to prevent acquisition of mature cell polarity (differentiation) until the taste bud achieves the appropriate size. In older fetal papillae with multiple taste buds, more punctate basement membrane disruptions could serve to permit continued access of nerves to cells in established taste buds. It is noteworthy that basement membrane disruptions are not observed in other papilla or lingual locations during development.

Because tenascin, which is a potential deadhesion molecule, is absent in the apex of gustatory papillae during initial taste bud development, disruptions in localization of a key adhesion molecule, laminin, may be necessary; this would permit taste bud cells to aggregate and form an immature taste bud. The absent or weak tenascin localization may be necessary for early innervation of the 50DF papillae and taste buds. Through distinctive temporal patterns, extracellular matrix molecules may fill various roles that permit and direct gustatory morphogenesis.

Like other extracellular matrix molecules, roles for laminin in diverse cell behaviors have been proposed during the development of various tissues, and the different roles are presumably mediated by multiple subunit chains that are present in several isoforms of the molecule (Tryggvason, 1993). For example, molecular isoforms that are apparently specific to epithelial and mesenchymal cells have been identified, and, presumably, laminin has cell-specific functions in these different tissue compartments. Similarly, we expect different functions and varying isoforms for laminin immunolocalized to epithelial basement membrane, nerves, 
and components of the mesenchyme in the developing tongue and papillae.

\section{GAP-43 immunoreactivity in developing papillae and taste buds}

At 50DF, when taste buds are first forming, GAP-43 immunoreactivity fills much of the core of the early fungiform and circumvallate papillae. The nature of the immunoproduct is rather diffuse at 50DF, and it alters to become more fiber-like at $80 \mathrm{DF}$ through $100 \mathrm{DF}$, when distinct bundles and small nerve branches are apparent within the papillae. Because GAP-43 is also present in Schwann cell precursors and in mature nonmyelin-forming Schwann cells in peripheral nerve (Curtis et al., 1992), it is possible that the early, diffuse immunoreaction reflects inclusion of immature Schwann cell elements as well as neurons.

Throughout development, GAP-43 immunoreactivity is most extensive and intense in papilla regions where tenascin is absent or weak. For example, the early fungiform papilla core is virtually devoid of tenascin immunoreactivity but is replete with GAP-43 label. At later stages (>110DF), the large GAP-43-positive nerve bundle at the middle base of the papilla travels in a tenascin-free region. In the apical papilla core, where tenascin label is organized in a lacework, neural elements are organized in a fine network.

The contrasting presence and intensity of tenascin and GAP-43 immunoreactivities support the proposed role for tenascin in nerve fasciculation (Wehrle and Chiquet, 1990). Recent experiments suggest a combined role of tenascin in peripheral nerve outgrowth in permitting axon growth but restricting satellite cell migration into nearby mesenchyme, thereby promoting fasciculation (Wehrle-Haller and Chiquet, 1993).

Another distinctive characteristic of GAP-43 immunoreactivity is the progression that takes place from 50DF to $110 \mathrm{DF}$ in the epithelium of both fungiform and circumvallate papillae. GAP-43 initially has a limited distribution in epithelial regions, where the large presumptive taste bud is forming, but is eventually extensive within each individual taste bud. Electron microscopic examination of taste buds in fungiform papillae of fetal sheep at 80DF demonstrates that there are numerous nerve profiles throughout the taste bud at this age (Mistretta and Bradley, 1983a); earlier ages were not examined. Therefore, the extensive GAP-43 label within the developing taste bud from 80DF to 100DF and beyond is associated temporally with a direct demonstration of extensive innervation. The GAP-43 immunoreactivity within developing taste buds presumably reflects synaptogenesis between gustatory nerves and taste bud cells. When recording from the chorda tympani (Bradley and Mistretta, 1973; Mistretta and Bradley, 1983a) and glossopharyngeal (Mistretta and Bradley, 1983b) nerves that innervate taste buds in fungiform and circumvallate papillae, respectively, neural responses have not been obtained before about $100 \mathrm{DF}$, and only very small magnitude responses are recorded at 100DF. By 110DF, larger magnitude, more reproducible neurophysiological responses are recorded, although the responses are still very immature in the nature and range of chemicals that elicit a response. There is a continued maturation in taste response magnitudes and characteristics after 110DF and throughout the postnatal period in sheep.

GAP-43 is a membrane phosphoprotein that is synthesized by developing neurons, transported to the growing axons, and concentrated in axonal growth cones (Gordon-
Weeks, 1989; Curtis et al., 1992). The protein is thought to participate in synaptogenesis, and levels of immunoreactivity decline in other systems after the respective target is innervated (Coggeshall et al., 1991; Reynolds et al., 1991). The time course of changes in GAP-43 immunolocalization in developing papillae and taste buds strongly suggests that this protein is associated not only with synaptogenesis per se, as discussed in the preceding paragraph, but is also associated with papilla and taste bud innervation during periods of receptive-field remodeling. GAP-43 immunoreactivity remains intense in fungiform papillae and taste buds at $130 \mathrm{DF}$ and perinatal stages, periods when the size and response properties of receptive fields of peripheral taste nerves are changing (Mistretta et al., 1988; Nagai et al., 1988). In adult papillae, however, GAP-43 immunoreactivity cannot be demonstrated, whereas immunolabel for neurofilament ( $200 \mathrm{kDa}$ protein subunit) remains in gustatory papillae in adult sheep (work in progress). Although taste bud cells continue to turn over, therefore breaking and reestablishing axonal contacts in adult mammals (Beidler and Smallman, 1965), this activity is much reduced compared to axon growth and terminal arbor extension during the actual establishment of a functional taste periphery. Therefore, GAP-43, in fact, might label gustatory nerve terminals only during periods of functional differentiation.

The immunocytochemical localization of cell adhesion molecules in the peripheral taste system has been described, and possible roles for the molecules in synaptogenesis in the taste bud have been proposed. For example, neural cell adhesion molecule (N-CAM) is immunolocalized in embryonic mouse papilla epithelium in regions where taste buds will later develop; N-CAM appearance is in temporal concert with the observation of extensive epithelial innervation (Nolte and Martini, 1992). In adult rat circumvallate papilla, various isoforms of N-CAM are located on taste bud cells (Nelson and Finger, 1993; Smith et al., 1993). Transection of gustatory nerves eliminates the N-CAM immunoreactivity in taste buds and in gustatory papillae (Smith et al., 1993). It has been suggested that $\mathrm{N}$-CAM localization on taste bud cells might indicate sights of synapse formation or specialized contact (Nelson and Finger, 1993; Smith et al., 1994).

\section{CONCLUSIONS}

In summary, immunocytochemical distributions of tenascin and laminin provide strong evidence that these extracellular matrix molecules participate in gustatory papilla and taste bud morphogenesis and that they possibly interact with papilla innervation during periods of extensive terminal fiber growth and branching. Tenascin and laminin have been contrasted as deadhesion and adhesion molecules, respectively (Murphy-Ullrich et al., 1991), and their temporal and spatial patterns of immunoreactivity provide support for the proposition that such functions may obtain during gustatory papilla development. These results add a new dimension to the consideration of taste organ development: that is, a potentially active regulatory role for the extracellular matrix.

\section{ACKNOWLEDGMENTS}

We thank Dr. Dennis Lopatin, School of Dentistry, for immunologic analysis of the tenascin antibody. This research was supported by grant DC00456 from the National 
Institute on Deafness and Other Communication Disorders (NIH) to C.M.M.

\section{LITERATURE CITED}

Adams, J.C., and F.M. Watt (1993) Regulation of development and differentiation by the extracellular matrix. Development 117:1183-1198.

Aufderheide, E., R. Chiquet-Ehrismann, and P. Ekblom (1987) Epithelialmesenchymal interactions in the developing kidney lead to expression of tenascin in the mesenchyme. J. Cell Biol. 105:599-608.

Beck, K., I. Hunter, and J. Engel (1990) Structure and function of laminin: Anatomy of a multidomain glycoprotein. FASEB J. 4:148-160.

Beidler, L.M., and R.L. Smallman (1965) Renewal of cells within taste buds. J. Cell Biol. 27:263-272.

Bradley, R.M., and C.M. Mistretta (1973) The gustatory sense in foetal sheep during the last third of gestation. J. Physiol. 231:271-282.

Chen, W.-T. (1992) Membrane proteases: Roles in tissue and tumour invasion. Curr. Opin. Biol. 4:802-809

Chiquet, M. (1989) Tenascin/J1/cytotactin: The potential function of hexabrachion proteins in neural development. Dev. Neurosci. 11:266275.

Chiquet-Ehrismann, R. (1990) What distinguishes tenascin from fibronectin? FASEB J. 4:2598-2604.

Chiquet-Ehrismann, R., Y. Matsuoka, U. Hofer, J. Spring, C. Bernasconi, and $M$. Chiquet (1991) Tenascin variants: Differential binding to fibronectin and distinet distribution in cell cultures and tissues. Cell Regul 2:927-938

Clark, P., S. Britland, and P. Connolly (1993) Growth cone guidance and neuron morphology on micropatterned laminin surfaces. J. Cell Sci 105:203-212

Coggeshall, R.E., M.L. Reynolds, and C.J. Woolf (1991) Distribution of the growth associated protein GAP-43 in the central processes of axotomized primary afferents in the adult rat spinal cord: Presence of growth cone-like structures. Neurosei. Lett. 131:37-41.

Curtis, R., H.J.S. Stewart, S.M. Hall, G.P. Wilkin, R. Mirsky, and K.R Jessen (1992) GAP-43 is expressed by nonmyelin-forming Schwann cells of the peripheral nervous system. J. Cell Biol. 116:1455-1464.

Ekblom, P. (1989) Developmentally regulated conversion of mesenchyme to epithelium. FASEB J. 3:2141-2150

End, P., and J. Engel (1991) Multidomain proteins of the extracellular matrix and cellular growth. In J.A. McDonald and R.P. Mecham (eds.). Receptors for Extracellular Matrix. New York: Academic Press, pp. $79-129$.

Erickson, H.P. (1993) Tenascin-C, tenascin-R and tenascin-X: A family of talented proteins in search of functions. Curr. Opin. Cell Biol. 5:869 876.

Erickson, H.P., and V.A. Lightner (1988) Hexabrachion protein (tenascin cytotactin, brachionectin) in connective tissue, embryonic brain and tumors. In K.R. Miller (ed.): Advances in Cell Biology, Vol. 2. London: JAI, pp. 55-90.

Form, D.M., B.M. Pratt, and J.A. Madri (1986) Endothelial cell proliferation during angiogenesis. In vitro modulation by basement membrane cell components. Lab. Invest. 55:521-530.

Gordon-Weeks, P.R. (1989) GAP-43-What does it do in the growth cone Trends Neurosci. 12:363-365.

Haus, L.F., and C.M. Mistretta (1992) Changes in distribution of tenascin correlate with gustatory papilla and taste bud development and morphogenesis in fetal sheep. Chem. Senses 17:498

Julian, J., R. Chiquet-Ehrismann, H.P. Erickson, and D.D. Carson (1994) Tenascin is induced at implantation sites in the mouse uterus and interferes with epithelial cell adhesion. Development 120:661-671.

Kaplan, E.D., and K.A. Holbrook (1994) Dynamic expression patterns of tenascin, proteoglycans, and cell adhesion molecules during human hair follicle morphogenesis. Dev. Dynam. 199:141-155.

Klein, G., M. Langegger, R. Timpl, and P. Ekblom (1988) Role of laminin A chain in the development of epithelial cell polarity. Cell 55:331-341.

Kleinman, H.K., F.B. Cannon, G.W. Laurie, J.R. Hassell, M. Aumailley, V.P. Terranova, G.R. Martin, and M. DuBois-Dalcq (1985) Biological activi ties of laminin. J. Cell. Biochem. 27:317-325.

Koch, M., B. Wehrle-Haller, S. Baumgartner, J. Spring, D. Brubacher, and M. Chiquet (1991) Epithelial synthesis of tenascin at tips of growing bronchi and graded accumulation in basement membrane and mesenchyme. Exp. Cell Res. 194:297-300.
Lightner, V.A., F. Gumkowski, D.D. Bigner, and H.P. Erickson (1989) Tenascin/hexabrachion in human skin: Biochemical identification and localization by light and electron microscopy. J. Cell Biol. 108:24832493.

Martin, G.R., and R. Timpl (1987) Laminin and other basement membrane components. Annu. Rev. Cell Biol. 3:57-85.

Mistretta, C.M. (1990) Taste development. In J.R. Coleman (ed.): Development of Sensory Systems in Mammals. New York: Wiley Interscience, pp. $567-613$.

Mistretta, C.M. (1991) Developmental neurobiology of taste. In T. Getchell, R. Doty, L. Bartoshuk, and J. Snow (eds.): Smell and Taste in Health and Disease. New York: Raven Press, pp. 35-64.

Mistretta, C.M. (1993) Developmental remodeling of peripheral and central taste receptive fields and circuits. In J. Pearl (ed.): Development, Growth and Senescence in the Chemical Senses, NIDCD Monograph. Bethesda MD: NIH Publication No. 93-3483, pp. 31-39.

Mistretta, C.M., and R.M. Bradley (1983a) Neural basis of developing salt taste sensation: Response changes in fetal, postnatal and adult sheep. $J$ Comp. Neurol. 215:199-210.

Mistretta, C.M., and R.M. Bradley (1983b) Developmental changes in taste responses from glossopharyngeal nerve in sheep and comparisons with chorda tympani responses. Dev. Brain Res. 11:107-117.

Mistretta, C.M., and L.F. Haus (1992) Tenascin distribution during development of taste organs suggests roles in regulating taste bud multiplication and papilla innervation. Soc. Neurosci. Abstr. 18:619.

Mistretta, C.M, and L.F. Haus (1993) Extracellular matrix molecules may modulate cell adhesion during papilla and taste bud morphogenesis and innervation. Chem. Senses 18:601.

Mistretta, C.M., and D.L. Hill (1995) Development of the taste system: Basic neurobiology. In R.L. Doty (ed.): Handbook of Olfaction and Gustation. New York: Dekker, pp. 635-668.

Mistretta, C.M., and S. Labyak (1994) Maturation of neuron types in nucleus of solitary tract associated with functional convergence during development of taste circuits. J. Comp. Neurol. 345:359-376.

Mistretta, C.M., S. Gurkan, and R.M. Bradley (1988) Morphology of chorda tympani fiber receptive fields and proposed neural rearrangements during development. J. Neurosci. 8:73-78

Morris-Wiman, J., and L. Brinkley (1994) The distribution of tenascin and syndecan during fungiform papilla morphogenesis. Association for Chemoreception Sciences: published in Chemical Senses 19:524.

Murphy-Ullrich, J.E., V.A. Lightner, I. Aukhil, Y.Z. Yan, H.P. Erickson, and M. Hook (1991) Focal adhesion integrity is downregulated by the alternatively spliced domain of human tenascin. J. Cell Biol. 115:11271136

Nagai, T., C.M. Mistretta, and R.M. Bradley (1988) Developmental decrease in size of peripheral receptive fields of single chorda tympani nerve fibers and relation to increasing $\mathrm{NaCl}$ taste sensitivity. J Neurosei. 8:64-72.

Nelson, G.M., and T.E. Finger (1993) Immunolocalization of different forms of neural cell adhesion molecule (NCAM) in rat taste buds. J. Comp Neurol. 336:507-516.

Nolte, C., and R. Martini (1992) Immunocytochemical localization of the L1 and N-CAM cell adhesion molecules and their shared carbohydrate epitope L2/HNK-1 in the developing and differentiated gustatory papillae of the mouse tongue. J. Neurocytol. 21:19-33.

Rettig, W.J., S. Hoffman, S.L. Su, and P. Garin-Chesa (1992) Species diversity of neuronectin and cytotactin expression patterns in the vertebrate central nervous system. Brain Res. 590:219-228.

Rettig, W.J., H.P. Erickson, A.P. Albino, and P. Garin-Chesa (1994) Induction of human tenascin (neuronectin) by growth factors and cytokines: Cell type-specific signals and signalling pathways. J. Cell Sci. 107:487-497.

Reynolds, M.L., M. Fitzgerald, and L.I. Benowitz (1991) GAP-43 expression in developing cutaneous and muscle nerves in the rat hindlimb. Neuroscience 41:201-211.

Riggott, M.J., and S.A. Moody (1987) Distribution of laminin and fibronectin along peripheral trigeminal axon pathways in the developing chick. $J$. Comp. Neurol. 259:580-596.

Rogers, S.L., K.J. Edson, P.C. Letourneau, and S.C. McLoon (1986) Distribution of laminin in the developing peripheral nervous system of the chick. Dev. Biol. 113:429-435

Saga, Y., T. Yagi, Y. Ikawa, T. Sakakura, and S. Aizawa (1992) Mice develop normally without tenascin. Genes Dev. 6:1821-1831.

Smith, D.V., R.A. Akeson, and M.T. Shipley (1993) NCAM expression by 
subsets of taste cells is dependent upon innervation. J. Comp. Neurol. 336:493-506.

Smith, D.V., R. Klevitsky, R.A. Akeson, and M.T. Shipley (1994) Expression of the neural cell adhesion molecule (NCAM) and polysialic acid during taste bud degeneration and regeneration. J. Comp. Neurol. 347:187-196

Spring, J. K Beck, and R. Chiquet-Ehrismann (1989) Two contrary functions of tenascin: Dissection of the active sites by recombinan tenascin fragments. Cell 59:325-334.

Thesleff, I., E. Mackie, S. Vianio, and R. Chiquet-Ehrismann (1987) Changes in the distribution of tenascin during tooth development. Development $101: 289-296$

Tomaselli, K.J., P. Doherty, C.J. Emmett, C.H. Damsky, F.S. Walsh, and L.F. Reichardt (1993) Expression of b1 integrins in sensory neurons of the dorsal root ganglion and their functions in neurite outgrowth on two laminin isoforms. J. Neurosci. 13:4880-4888.

Trembie, P., R. Chiquet-Ehrismann, and Z. Werb (1994) The extracellular matrix ligands fibronectin and tenascin collaborate in regulating collagenase gene expression in fibroblasts. Mol. Biol. Cell 5:439-453.

Tryggvason, K. (1993) The laminin family. Current Opin. Cell Biol. 5:877882.

Tucker, R.P. (1991) The sequential expression of tenascin mRNA in epithelium and mesenchyme during feather morphogenesis. Dev. Biol. $200 \cdot 108-112$.
Tucker, R.P. J. Spring, S Baumgartner, D Martin C. Hagios, P.M. Poss, and $R$. Chiquet-Ehrismann (1994) Novel tenascin variants with a distinctive pattern of expression in the avian embryo. Development 120:637-647.

Van Duinen, C.M., G.J. Fleuren, and J.A. Bruijn (1994) The extracellular matrix in pigmented skin lesions: An immunohistochemical study. Histopathology 24:33-40.

Vogt, M., and C.M. Mistretta (1990) Convergence in mammalian nucleus of solitary tract during development and differentiation of salt taste circuits. J. Neurosci. 10:3148-3157.

Wehrle, B., and M. Chiquet (1990) Tenascin is accumulated along developing peripheral nerves and allows neurite outgrowth in vitro. Development 110:401-415.

Wehrle-Haller, B., and M. Chiquet (1993) Dual function of tenascin: Simultaneous promotion of neurite growth and inhibition of glial migration. J. Cell Sci. 106:597-610.

Woodley, D.T., P.M. Bachmann, and E.J. O'Keefe (1988) Laminin inhibits human keratinocyte migration. J. Cell Physiol. 136:140-146.

Young, S.L., L.-Y. Chang, and H.P. Erickson (1994) Tenascin-C in rat lung: Distribution, ontogeny and role in branching morphogenesis. Dev. Biol. 161:615-625.

Yurchenco, P.D., and J.C. Schittny (1990) Molecular architecture of basement membranes. FASEB J. 4:1577-1590. 